\title{
Maximal Functions Along Convex Curves with Lacunary Directions
}

\section{Haixia Yu}

Abstract. In this paper, we obtain the $L^{p}\left(\mathbb{R}^{2}\right)$-boundedness of the maximal functions

$$
M_{I, \gamma} f\left(x_{1}, x_{2}\right):=\sup _{j \in \mathbb{Z}} \sup _{\varepsilon>0} \frac{1}{2 \varepsilon} \int_{-\varepsilon}^{\varepsilon}\left|f\left(x_{1}-t, x_{2}-2^{j} \gamma(t)\right)\right| \mathrm{d} t
$$

and

$$
M_{I I, \gamma} f\left(x_{1}, x_{2}\right):=\sup _{i, j \in \mathbb{Z}} \sup _{\varepsilon>0} \frac{1}{2 \varepsilon} \int_{-\varepsilon}^{\varepsilon}\left|f\left(x_{1}-2^{i} t, x_{2}-2^{j} \gamma(t)\right)\right| \mathrm{d} t,
$$

where $p \in(1, \infty]$ and $\gamma$ is a convex curve satisfying some suitable curvature conditions.

\section{Introduction}

The maximal function $M_{\gamma}$ along the curve $(t, \gamma(t))$ is defined by

$$
M_{\gamma} f\left(x_{1}, x_{2}\right):=\sup _{\varepsilon>0} \frac{1}{2 \varepsilon} \int_{-\varepsilon}^{\varepsilon}\left|f\left(x_{1}-t, x_{2}-\gamma(t)\right)\right| \mathrm{d} t
$$

The question of whether this operator $M_{\gamma}$ is bounded on $L^{p}\left(\mathbb{R}^{2}\right)$ has received much attention in the last few decades. In particular, for $\gamma(t):=t^{2}$, Nagel, Riviere and Wainger 22 ] established the $L^{p}\left(\mathbb{R}^{2}\right)$-boundedness of $M_{\gamma}$ for all $p \in(1, \infty]$. For the cases of homogeneous curves and smooth curves, we refer to Stein [27] and Stein and Wainger [28], respectively. Later, Stein and Wainger $\left[29\right.$ obtained the $L^{p}\left(\mathbb{R}^{2}\right)$-boundedness, $p \in(1, \infty]$, of $M_{\gamma}$ for "well-curved" curves, they also pointed out that this boundedness is false for arbitrary $C^{\infty}$ curves. Therefore, for more general curves obeying some curvature conditions, obtaining the $L^{p}\left(\mathbb{R}^{2}\right)$-boundedness of the associated maximal function $M_{\gamma}$ has become a classical problem in harmonic analysis.

For the case of $\gamma \in C^{2}(\mathbb{R})$ is either odd or even, $\gamma(0)=\gamma^{\prime}(0)=0$, and convex on $(0, \infty)$, let $h(t):=t \gamma^{\prime}(t)-\gamma(t)$, if $h$ satisfies the doubling condition, i.e.,

(D) There exists $1<\varepsilon_{0}<\infty$ so that $h\left(\varepsilon_{0} t\right) \geq 2 h(t)$ for all $0<t<\infty$,

Received January 27, 2021; Accepted December 22, 2021.

Communicated by Sanghyuk Lee.

2020 Mathematics Subject Classification. 42B25.

Key words and phrases. maximal function along curve, Littlewood-Paley operator, oscillatory integral. The author is supported by STU Scientific Research Foundation for Talents (No. NTF21038) and Guangdong Basic and Applied Basic Research Foundation (No. 2020A1515110241). 
Nagel, Vance, Wainger and Weinberg 25 showed the $L^{2}\left(\mathbb{R}^{2}\right)$-boundedness of $M_{\gamma}$. Later, Carlsson, Christ, Córdoba et al. [7] extended this boundedness to all $p \in(1, \infty]$ if $\gamma^{\prime}$ satisfies the doubling condition (D). Furthermore, Carbery, Christ, Vance et al. [4] also obtained the $L^{p}\left(\mathbb{R}^{2}\right)$-boundedness of $M_{\gamma}$ for all $p \in(1, \infty]$ by replacing this doubling condition (D) about $\gamma^{\prime}$ with the following infinitesimally doubling condition about $h$, i.e.,

$$
\text { There exists } 0<\varepsilon_{1}<\infty \text { so that } h^{\prime}(t) \geq \varepsilon_{1} \frac{h(t)}{t} \text { for all } 0<t<\infty \text {. }
$$

Note that both of $\gamma^{\prime}$ satisfies the doubling condition (D) and $h$ satisfies the infinitesimally doubling condition (ID) imply that $h$ satisfies the doubling condition (D). For more discussion in the case of $\gamma$ is convex, we refer to [8, 10,33], but a necessary and sufficient condition for the $L^{p}\left(\mathbb{R}^{2}\right)$-boundedness of $M_{\gamma}$ is still not known. There are many other cases of the curves $\gamma$ in obtaining the $L^{p}\left(\mathbb{R}^{2}\right)$-boundedness of $M_{\gamma}$; see, for example, nonconvex curves [1,31], flat curves [5, 6, highly monotone curves [26], oscillating curves [32]. The immediate consequence of the $L^{p}\left(\mathbb{R}^{2}\right)$-boundedness of $M_{\gamma}$ is obtaining the Lebesgue differentiation theorem along the curve $(t, \gamma(t))$, i.e., for any $f \in L^{p}\left(\mathbb{R}^{2}\right)$,

$$
\lim _{\varepsilon \rightarrow 0} \frac{1}{2 \varepsilon} \int_{-\varepsilon}^{\varepsilon} f\left(x_{1}-t, x_{2}-\gamma(t)\right) \mathrm{d} t=f\left(x_{1}, x_{2}\right) \quad \text { a.e. }\left(x_{1}, x_{2}\right) \in \mathbb{R}^{2} .
$$

In this paper, we consider two different kind of maximal functions analogue of $M_{\gamma}$, which are modelled on the maximal function $M_{\gamma}$ in lacunary direction introduced by Nagel, Stein and Wainger in [23]. The first one is the maximal function $M_{I, \gamma}$ along the curve $(t, \gamma(t))$ with the dyadic lacunary direction $\left(1,2^{j}\right)$,

$$
M_{I, \gamma} f\left(x_{1}, x_{2}\right):=\sup _{j \in \mathbb{Z}} \sup _{\varepsilon>0} \frac{1}{2 \varepsilon} \int_{-\varepsilon}^{\varepsilon}\left|f\left(x_{1}-t, x_{2}-2^{j} \gamma(t)\right)\right| \mathrm{d} t .
$$

The second one is the maximal function $M_{I I, \gamma}$ along the curve $(t, \gamma(t))$ with the dyadic lacunary direction $\left(2^{i}, 2^{j}\right)$, which allow dyadic scaling along this dyadic lacunary direction $\left(1,2^{j}\right)$ in $M_{I, \gamma}$,

$$
M_{I I, \gamma} f\left(x_{1}, x_{2}\right):=\sup _{i, j \in \mathbb{Z}} \sup _{\varepsilon>0} \frac{1}{2 \varepsilon} \int_{-\varepsilon}^{\varepsilon}\left|f\left(x_{1}-2^{i} t, x_{2}-2^{j} \gamma(t)\right)\right| \mathrm{d} t .
$$

Remark 1.1. Indeed, both of $M_{I, \gamma}$ and $M_{I I, \gamma}$ are a special case of the following maximal function $\mathcal{M}_{I I, \gamma}$ along the curve $(t, \gamma(t))$ with the direction $(a, b)$,

$$
\mathcal{M}_{I I, \gamma} f\left(x_{1}, x_{2}\right):=\sup _{a, b \in \mathbb{R}} \sup _{\varepsilon>0} \frac{1}{2 \varepsilon} \int_{-\varepsilon}^{\varepsilon}\left|f\left(x_{1}-a t, x_{2}-b \gamma(t)\right)\right| \mathrm{d} t .
$$

Note that $\mathcal{M}_{I I, \gamma}$ arise in connection with Stein's and Bourgain's circular maximal function. In [21], Marletta and Ricci obtained that $\mathcal{M}_{I I, \gamma}$ is bounded on $L^{p}\left(\mathbb{R}^{2}\right)$ if and only if 
$p \in(2, \infty]$, where $\gamma(t):=t^{r}$ with $r \in \mathbb{R}$ and $r \neq 0,1$. If $\gamma(t):=t$, a counter-example based on a construction of the Besicovitch-Kakeya set shows that we can not expect any $L^{p}\left(\mathbb{R}^{2}\right)$-boundednesss of $\mathcal{M}_{I I, \gamma}$ for all $p \in(1, \infty)$. More related works can be found in Bourgain [2], Iosevich [16], Marletta [20, Guo, Hickman, Lie and Roos [12] and Li [19]. Here, we also want to introduce another operator, which is closely related to $\mathcal{M}_{I I, \gamma}$, i.e., the corresponding maximal Hilbert transform $\mathcal{H}_{I I, \gamma}$ along the curve $(t, \gamma(t))$ with the direction $(a, b)$,

$$
\mathcal{H}_{I I, \gamma} f\left(x_{1}, x_{2}\right):=\sup _{a, b \in \mathbb{R}}\left|\mathrm{P} . \mathrm{V} \cdot \int_{-\infty}^{\infty} f\left(x_{1}-a t, x_{2}-b \gamma(t)\right) \frac{\mathrm{d} t}{t}\right| .
$$

From a straightforward modification of Karagulyan's counter-example in [17], we will find that the $L^{p}\left(\mathbb{R}^{2}\right)$-boundedness of $\mathcal{H}_{I I, \gamma}$ fails for all $p \in(1, \infty]$ even in the case of $\gamma(t):=t^{2}$ or $\gamma(t):=t$. Therefore, instead of considering the $L^{p}\left(\mathbb{R}^{2}\right)$-boundedness of $\mathcal{H}_{I I, \gamma}$, we always consider a new operator $\mathcal{H}_{U, \gamma}$ constructed by replacing $\sup _{a, b \in \mathbb{R}}$ with $\sup _{a, b \in U}$ in $\mathcal{H}_{I I, \gamma}$, and calculate the operator norm $\left\|\mathcal{H}_{U, \gamma}\right\|_{L^{p}\left(\mathbb{R}^{2}\right) \rightarrow L^{p}\left(\mathbb{R}^{2}\right)}$, where $U \subset \mathbb{R}$ is an arbitrary nonempty set. This is also a hot topic in harmonic analysis, see Demeter and Di Plinio [11], Laba, Marinelli and Pramanik [18], Guo, Roos, Seeger and Yung [13 and the references therein. In this paper, we are not going to calculate the $L^{p}\left(\mathbb{R}^{2}\right)$-norm of this maximal singular integral operator $\mathcal{H}_{U, \gamma}$, and our results of the $L^{p}\left(\mathbb{R}^{2}\right)$-boundedness of $M_{I, \gamma}$ and $M_{I I, \gamma}$ will provide an inspiration for obtaining the $L^{p}\left(\mathbb{R}^{2}\right)$-boundedness of $\mathcal{M}_{I I, \gamma}$ for some $p \in(1, \infty)$ and more general class of curves $\gamma$.

We now state our results.

Theorem 1.2. Let $\gamma \in C^{2}(\mathbb{R})$ be either odd or even, $\gamma(0)=\gamma^{\prime}(0)=0$, and convex on $(0, \infty)$, and satisfying

(i) there exists a positive constant $C_{1}$ such that $\frac{\gamma^{\prime}(2 t)}{\gamma^{\prime}(t)} \leq C_{1}$ for any $t \in(0, \infty)$;

(ii) there exists a positive constant $C_{2}$ such that $\frac{t \gamma^{\prime \prime}(t)}{\gamma^{\prime}(t)} \geq C_{2}$ for any $t \in(0, \infty)$.

Then for any given $p \in(1, \infty]$, there exists a positive constant $C$ such that

$$
\left\|M_{I, \gamma} f\right\|_{L^{p}\left(\mathbb{R}^{2}\right)} \leq C\|f\|_{L^{p}\left(\mathbb{R}^{2}\right)}
$$

for any $f \in L^{p}\left(\mathbb{R}^{2}\right)$.

Theorem 1.3. Let $\gamma \in C^{2}(\mathbb{R})$ be either odd or even, $\gamma(0)=\gamma^{\prime}(0)=0$, and convex on $(0, \infty)$, and satisfying

(i) there exists a positive constant $C_{1}^{\prime}$ such that $\frac{t \gamma^{\prime}(t)}{\gamma(t)} \leq C_{1}^{\prime}$ for any $t \in(0, \infty)$;

(ii) there exists a positive constant $C_{2}$ such that $\frac{t \gamma^{\prime \prime}(t)}{\gamma^{\prime}(t)} \geq C_{2}$ for any $t \in(0, \infty)$. 
Then for any given $p \in(1, \infty]$, there exists a positive constant $C$ such that

$$
\left\|M_{I I, \gamma} f\right\|_{L^{p}\left(\mathbb{R}^{2}\right)} \leq C\|f\|_{L^{p}\left(\mathbb{R}^{2}\right)}
$$

for any $f \in L^{p}\left(\mathbb{R}^{2}\right)$.

Remark 1.4. The conditions of Theorem 1.2 imply the conditions of Theorem 1.3. Indeed, from (2.1), as in 24, Lemma 2], we have

$$
\frac{\gamma^{\prime}(2 t)}{\gamma^{\prime}(t)} \geq e^{C_{2} / 2} \quad \Longrightarrow \quad h(t) \geq \lambda t \gamma^{\prime}(t) \quad \Longrightarrow \quad h\left(\frac{2}{\lambda} t\right) \geq 2 h(t) \quad \text { for any } t \in(0, \infty) \text {, }
$$

where $h(t)=t \gamma^{\prime}(t)-\gamma(t)$ and $\lambda:=\frac{1-e^{-C_{2} / 2}}{2}$. Furthermore, as in 24, Lemma 1], we have

$$
\begin{aligned}
h\left(\frac{2}{\lambda} t\right) \geq 2 h(t) & \Longrightarrow \frac{2}{\lambda} t\left(\gamma^{\prime}(s)-\gamma^{\prime}(t)\right) \geq h(t) \text { with } s \geq \frac{2}{\lambda} t \\
& \Longrightarrow \gamma\left(\frac{4}{\lambda} t\right) \geq \frac{4}{\lambda} t \gamma^{\prime}(t) \text { for any } t \in(0, \infty)
\end{aligned}
$$

This, combined with Theorem $1.2(i)$, implies that $\frac{t \gamma^{\prime}(t)}{\gamma(t)} \leq\left(C_{1}\right)^{\log _{2} \frac{4}{\lambda}}$ for any $t \in(0, \infty)$. Therefore, we obtain Theorem $1.3(\mathrm{i})$ with $C_{1}^{\prime}:=\left(C_{1}\right)^{\log _{2} \frac{4}{\lambda}}$.

From this fact, it is easy to see that Theorem 1.2 can be covered by Theorem 1.3 . However, the method of proving Theorem 1.2 is totally different from the method applied in proving Theorem 1.3 . Therefore, we will retain Theorem 1.2

Example 1.5. Let us list some examples of curves satisfying the conditions in Theorems 1.2 and 1.3 . Since $\gamma(t)$ is odd or even, and $\gamma(0)=0$, we write only the part for $t>0$.

(1) for any $t>0, \gamma(t):=t^{\alpha}$, where $\alpha>1$;

(2) for any $k \in \mathbb{N}$ and $t>0, \gamma(t):=\sum_{i=1}^{k} t^{\alpha_{i}}$, where $\alpha_{i}>1$ for all $i=1,2, \ldots, k$;

(3) for any $t>0, \gamma(t):=t^{\alpha} \ln (1+t)$, or $\gamma(t):=t^{\alpha} \arctan t$, where $\alpha>1$;

(4) for any $t>0, \gamma(t):=(t \sin t) \mathbf{1}_{\left\{0<t<\varepsilon_{0}\right\}}(t)$, or $(t-\sin t) \mathbf{1}_{\left\{0<t<\varepsilon_{0}\right\}}(t)$, or $(t-\ln (1+$ $t)) \mathbf{1}_{\left\{0<t<\varepsilon_{0}\right\}}(t)$, where $\varepsilon_{0}$ is small enough.

It is easy to see that, for the case of $\gamma(t):=t^{d}$ under $d \in \mathbb{N}$, the $L^{p}\left(\mathbb{R}^{2}\right)$-boundedness of $M_{I, \gamma}$ and $M_{I I, \gamma}$ are equivalent. But for more general curves, this equivalence relation does not hold. Therefore, we consider $M_{I, \gamma}$ and $M_{I I, \gamma}$, respectively. The history of these operators goes back to the case of $\gamma(t):=t$. Córdoba and Fefferman [9] and Strömberg [30 used a suitable geometric argument to obtain the $L^{p}\left(\mathbb{R}^{2}\right)$-boundedness of $M_{I I, \gamma}$ for all $p \in[2, \infty]$. Later, Nagel, Stein and Wainger [23] extended this result to all $p \in(1, \infty]$ 
by using a Littlewood-Paley decomposition. Furthermore, Carbery [3] extended these two-dimensional results [9, 23, 30] to arbitrary dimension. For the case of $\gamma(t):=t^{2}$, Guo, Roos, Seeger and Yung 13, Lemma 5.1] obtained the $L^{p}\left(\mathbb{R}^{2}\right)$-boundedness of $M_{I, \gamma}$ for all $p \in(1, \infty]$, and the same boundedness for the case of $\gamma(t):=[t]^{\alpha}$ can also been obtained with some standard modifications, where $[t]^{\alpha}$ stands for either $|t|^{\alpha}$ or $\operatorname{sgn}(t)|t|^{\alpha}, \alpha>0$ and $\alpha \neq 1$. There are also some other types of $M_{I I, \gamma}$; see, for example,

$$
M_{I I}^{Q} f\left(x_{1}, x_{2}\right):=\sup _{i, j \in \mathbb{Z}} \sup _{\varepsilon>0} \frac{1}{2 \varepsilon} \int_{-\varepsilon}^{\varepsilon}\left|f\left(x_{1}-2^{i} Q(t), x_{2}-2^{j} Q(t)\right)\right| \mathrm{d} t,
$$

where $Q$ is a real polynomial with $Q(0)=0$. Hare and Ricci 14 obtained the $L^{p}\left(\mathbb{R}^{2}\right)$ boundedness of $M_{I I}^{Q}$ for all $p \in(1, \infty]$. More recently, Hong, Kim and Yang [15] extended $M_{I I}^{Q}$ to more general polynomial curves and arbitrary dimension.

Our proofs of Theorems 1.2 and 1.3 rely on the non-stationary phase method in Carlsson, Christ, Córdoba et al. [7], the Littlewood-Paley theory, and a bootstrapping argument similar that of Nagel, Stein and Wainger 23. In Section 2, we prove Theorem 1.2 by comparing it with a smoother operator. Theorem 1.3 is proved in Section 3 , we split it as the low-frequency part and the high-frequency part. The low-frequency part can be viewed as a one-dimensional convolution type operator, and the high-frequency part is proved by interpolating between a decay estimate under $p \in[2, \infty)$ and a non-decay estimate under $p \in(1,2)$.

Throughout this paper, we use "C" to denote a positive constant that is independent of the main parameters involved but whose value may vary from line to line. The positive constants with subscripts, such as $C_{1}$ and $C_{2}$, are the same in different occurrences. For two real functions $f$ and $g$, we use $f \lesssim g$ or $g \gtrsim f$ to denote $f \leq C g$ and, if $f \lesssim g \lesssim f$, we then write $f \approx g$. We use $\mathbb{Z}$ to denote the set of integers, and $\mathbb{N}:=\{1,2,3, \ldots\}$. We also use $a \Rightarrow b$ to denote that $a$ implies $b$. For any set $E$, we use $\mathbf{1}_{E}$ to denote the characteristic function of $E$.

\section{Proof of Theorem 1.2}

We start by introducing some simple properties of the curve $\gamma$ which we need in the course of proof. Indeed, let $G(t):=\ln \gamma^{\prime}(t)$ for any $t \in(0, \infty)$, by Theorem $1.2($ ii), we then have $G^{\prime}(t) \geq \frac{C_{2}}{t}$ for any $t \in(0, \infty)$. By the Lagrange mean value theorem, there exists a constant $\theta \in[1,2]$ such that $G(2 t)-G(t)=G^{\prime}(\theta t) t \geq \frac{C_{2}}{2}$, which further leads to

$$
\frac{\gamma^{\prime}(2 t)}{\gamma^{\prime}(t)} \geq e^{C_{2} / 2} \quad \text { for any } t \in(0, \infty)
$$

On the other hand, since $\gamma(0)=\gamma^{\prime}(0)=0$, by the Cauchy mean value theorem, there exists $T \in(0, t)$ such that $\frac{t \gamma^{\prime}(t)}{\gamma(t)}=\frac{t \gamma^{\prime}(t)-0 \gamma^{\prime}(0)}{\gamma(t)-\gamma(0)}=\frac{\gamma^{\prime}(T)+T \gamma^{\prime \prime}(T)}{\gamma^{\prime}(T)}$ for any $t \in(0, \infty)$. Thus, we 
apply Theorem 1.2 (ii) to conclude that

$$
\frac{t \gamma^{\prime}(t)}{\gamma(t)} \geq C_{2}+1 \quad \text { for any } t \in(0, \infty) \text {. }
$$

We will make use of some ideas from Carlsson, Christ, Córdoba et al. [7] to prove our theorem. Indeed, it is naturally to consider the $L^{2}\left(\mathbb{R}^{2}\right)$-boundedness fristly, and also should control $\sup _{j, k \in \mathbb{Z}} M_{I, \gamma}^{j, k} f\left(x_{1}, x_{2}\right)$ by $\sum_{j, k \in \mathbb{Z}} M_{I, \gamma}^{j, k} f\left(x_{1}, x_{2}\right)$. From Plancherel's theorem, it is sufficient to bound the corresponding multiplier $\sum_{j, k \in \mathbb{Z}} m_{I, \gamma}^{j, k}\left(\xi_{1}, \xi_{2}\right)$. Furthermore, by applying van der Corput's lemma, we may bound the multiplier $m_{I, \gamma}^{j, k}\left(\xi_{1}, \xi_{2}\right)$ by $\left|\left(\varphi_{I, \gamma}^{j, k}\right)^{\prime}(t)\right|^{-1}$ or $\left|\left(\varphi_{I, \gamma}^{j, k}\right)^{\prime \prime}(t)\right|^{-1 / 2}$, where $\varphi_{I, \gamma}^{j, k}(t)$ is the corresponding phase function of the multiplier $m_{I, \gamma}^{j, k}\left(\xi_{1}, \xi_{2}\right)$, but it is not enough to bound $\sum_{j, k \in \mathbb{Z}} m_{I, \gamma}^{j, k}\left(\xi_{1}, \xi_{2}\right)$. Therefore, to bound this sum, we want to bound the multiplier $m_{I, \gamma}^{j, k}\left(\xi_{1}, \xi_{2}\right)$ by $\left|\varphi_{I, \gamma}^{j, k}(t)\right|$. Note that $\left|e^{i \varphi_{I, \gamma}^{j, k}(t)}-1\right| \leq\left|\varphi_{I, \gamma}^{j, k}(t)\right|$, this implies that we should define a new multiplier $\bar{m}_{I, \gamma}^{j, k}\left(\xi_{1}, \xi_{2}\right)$ to compare our multiplier $m_{I, \gamma}^{j, k}\left(\xi_{1}, \xi_{2}\right)$, it is also the reason of defining the operator $\sigma_{k}$ in 7. For the general $L^{p}\left(\mathbb{R}^{2}\right)$-boundedness, as in Nagel, Stein and Wainger [23], the bootstapping argument is a useful tool, and our proof also relies on this argument.

We now begin the proof of Theorem 1.2 . Since $M_{I, \gamma}$ is a positive operator we may assume that $f$ is non-negative. Furthermore, we can bound $M_{I, \gamma} f$ by

$$
\begin{aligned}
& \sup _{j \in \mathbb{Z}} \sup _{\varepsilon>0} \frac{1}{2 \varepsilon} \sum_{k: 2^{k} \leq \varepsilon} 2^{k} \frac{1}{2^{k}} \int_{2^{k}<|t| \leq 2^{k+1}} f\left(x_{1}-t, x_{2}-2^{j} \gamma(t)\right) \mathrm{d} t \\
& \lesssim \sup _{j, k \in \mathbb{Z}} \frac{1}{2^{k}} \int_{2^{k}<|t| \leq 2^{k+1}} f\left(x_{1}-t, x_{2}-2^{j} \gamma(t)\right) \mathrm{d} t=: \sup _{j, k \in \mathbb{Z}} M_{I, \gamma}^{j, k} f\left(x_{1}, x_{2}\right) .
\end{aligned}
$$

Therefore, it suffices to prove the $L^{p}\left(\mathbb{R}^{2}\right)$-boundedness of the operator $\sup _{j, k \in \mathbb{Z}} M_{I, \gamma}^{j, k}$. As in [7, we define a smoother operator $\bar{M}_{I, \gamma}^{j, k}$ by

$$
\bar{M}_{I, \gamma}^{j, k} f\left(x_{1}, x_{2}\right):=\frac{1}{2^{2 k}} \int_{2^{k}<|t| \leq 2^{k+1}} \int_{2^{k}<|\tau| \leq 2^{k+1}} f\left(x_{1}-t, x_{2}-2^{j} \gamma(\tau)\right) \mathrm{d} t \mathrm{~d} \tau,
$$

which keeps many the same characteristics from $M_{I, \gamma}^{j, k}$. More importantly, from 2.2 and the fact that $\left(\gamma^{-1}\right)^{\prime}(t) \gamma^{\prime}\left(\gamma^{-1}(t)\right)=1$ where $\gamma^{-1}$ is the inverse function of $\gamma$, by letting $s:=2^{j} \gamma(\tau)$, we can bound $\bar{M}_{I, \gamma}^{j, k} f\left(x_{1}, x_{2}\right)$ by

$$
\begin{aligned}
& \frac{1}{2^{2 k}} \int_{2^{k}<|t| \leq 2^{k+1}} \int_{2^{j} \gamma\left(2^{k}\right)<|s| \leq 2^{j} \gamma\left(2^{k+1}\right)} f\left(x_{1}-t, x_{2}-s\right)\left|\frac{\gamma\left(\gamma^{-1}\left(2^{-j} s\right)\right)}{\gamma^{\prime}\left(\gamma^{-1}\left(2^{-j} s\right)\right) \gamma^{-1}\left(2^{-j} s\right)}\right| \\
& \quad \times\left|\frac{\gamma^{-1}\left(2^{-j} s\right) 2^{-j}}{\gamma\left(\gamma^{-1}\left(2^{-j} s\right)\right)}\right| \mathrm{d} t \mathrm{~d} s .
\end{aligned}
$$

Furthermore, from 2.2 and Theorem $1.2(\mathrm{i})$, by simple calculation, one has

$$
\frac{1}{2^{2 k}} 2^{k+1} 2^{j} \gamma\left(2^{k+1}\right)\left|\frac{\gamma\left(\gamma^{-1}\left(2^{-j} s\right)\right)}{\gamma^{\prime}\left(\gamma^{-1}\left(2^{-j} s\right)\right) \gamma^{-1}\left(2^{-j} s\right)}\right| \cdot\left|\frac{\gamma^{-1}\left(2^{-j} s\right) 2^{-j}}{\gamma\left(\gamma^{-1}\left(2^{-j} s\right)\right)}\right| \lesssim 1,
$$


which further implies that $\bar{M}_{I, \gamma}^{j, k} f$ can be bounded by $M^{(1)} M^{(2)} f$. Here and hereafter, we use $M^{(1)}$ and $M^{(2)}$ denote the Hardy-Littlewood maximal operators applied in the first variable and the second variable, respectively. This implies the following pointwise estimate

$$
\sup _{j, k \in \mathbb{Z}} \bar{M}_{I, \gamma}^{j, k} f\left(x_{1}, x_{2}\right) \lesssim M^{(1)} M^{(2)} f\left(x_{1}, x_{2}\right) .
$$

From the $L^{p}\left(\mathbb{R}^{2}\right)$-boundedness of $M^{(1)}$ and $M^{(2)}$, we have

$$
\left\|\sup _{j, k \in \mathbb{Z}} \bar{M}_{I, \gamma}^{j, k} f\right\|_{L^{p}\left(\mathbb{R}^{2}\right)} \lesssim\left\|M^{(1)} M^{(2)} f\right\|_{L^{p}\left(\mathbb{R}^{2}\right)} \lesssim\|f\|_{L^{p}\left(\mathbb{R}^{2}\right)}
$$

for all $p \in(1, \infty]$. Therefore, it suffices to prove

$$
\left\|\sup _{j, k \in \mathbb{Z}}\left|\left(M_{I, \gamma}^{j, k}-\bar{M}_{I, \gamma}^{j, k}\right) f\right|\right\|_{L^{p}\left(\mathbb{R}^{2}\right)} \lesssim\|f\|_{L^{p}\left(\mathbb{R}^{2}\right)}
$$

for all $p \in(1, \infty]$.

\section{1. $L^{2}\left(\mathbb{R}^{2}\right)$-boundedness}

For $p=2$ in (2.4), it is naturally to consider the multipliers of $M_{I, \gamma}^{j, k}$ and $\bar{M}_{I, \gamma}^{j, k}$ by Plancherel's theorem. Indeed, a calculation gives that the multiplier of $M_{I, \gamma}^{j, k}$ can be written as

$$
m_{I, \gamma}^{j, k}\left(\xi_{1}, \xi_{2}\right):=\int_{1 \leq|t|<2} e^{-i 2^{k} t \xi_{1}-i 2^{j} \gamma\left(2^{k} t\right) \xi_{2}} \mathrm{~d} t
$$

and the multiplier of $\bar{M}_{I, \gamma}^{j, k}$ can be written as

$$
\bar{m}_{I, \gamma}^{j, k}\left(\xi_{1}, \xi_{2}\right):=\int_{1 \leq|t|<2} \int_{1 \leq|\tau|<2} e^{-i 2^{k} t \xi_{1}-i 2^{j} \gamma\left(2^{k} \tau\right) \xi_{2}} \mathrm{~d} t \mathrm{~d} \tau .
$$

We now bound the left-hand side of (2.4) by

$$
\left\|\left[\sum_{j, k \in \mathbb{Z}}\left|\left(M_{I, \gamma}^{j, k}-\bar{M}_{I, \gamma}^{j, k}\right) f\right|^{2}\right]^{1 / 2}\right\|_{L^{2}\left(\mathbb{R}^{2}\right)} .
$$

Because the $l^{2}\left(\mathbb{Z}^{2}\right)$ and $L^{2}\left(\mathbb{R}^{2}\right)$ norms commute, from Plancherel's theorem, it is enough to show that

$$
\sum_{j, k \in \mathbb{Z}}\left|m_{I, \gamma}^{j, k}\left(\xi_{1}, \xi_{2}\right)-\bar{m}_{I, \gamma}^{j, k}\left(\xi_{1}, \xi_{2}\right)\right|^{2} \lesssim 1
$$


for all $\left(\xi_{1}, \xi_{2}\right) \in \mathbb{R}^{2}$. Furthermore, let us set $\xi_{1}:=2^{M} \widetilde{\xi}_{1}$ and $\xi_{2}:=2^{N} \widetilde{\xi}_{2}$, where $M, N \in \mathbb{Z}$ and $\left|\widetilde{\xi}_{1}\right|,\left|\widetilde{\xi}_{2}\right| \in[1,2)$, then the left-hand side of $(2.5)$ is equal to

$$
\begin{aligned}
& \sum_{j, k \in \mathbb{Z}}\left|\int_{1 \leq|t|<2} e^{-i 2^{k+M} t \widetilde{\xi}_{1}-i 2^{j+N} \gamma\left(2^{k} t\right) \widetilde{\xi}_{2}} \mathrm{~d} t-\int_{1 \leq|t|<2} \int_{1 \leq|\tau|<2} e^{-i 2^{k+M} t \widetilde{\xi}_{1}-i 2^{j+N} \gamma\left(2^{k} \tau\right) \widetilde{\xi}_{2}} \mathrm{~d} t \mathrm{~d} \tau\right|^{2} \\
= & \sum_{j, k \in \mathbb{Z}}\left|\int_{1 \leq|t|<2} e^{-i 2^{k} t \widetilde{\xi}_{1}-i C_{M} 2^{j} \Gamma_{M}\left(2^{k} t\right) \widetilde{\xi}_{2}} \mathrm{~d} t-\int_{1 \leq|t|<2} \int_{1 \leq|\tau|<2} e^{-i 2^{k} t \widetilde{\xi}_{1}-i C_{M} 2^{j} \Gamma_{M}\left(2^{k} \tau\right) \widetilde{\xi}_{2}} \mathrm{~d} t \mathrm{~d} \tau\right|^{2} \\
= & : \sum_{j, k \in \mathbb{Z}}\left|\mathfrak{m}_{I, \gamma}^{j, k}\left(\widetilde{\xi}_{1}, \widetilde{\xi}_{2}\right)-\overline{\mathfrak{m}}_{I, \gamma}^{j, k}\left(\widetilde{\xi}_{1}, \widetilde{\xi}_{2}\right)\right|^{2},
\end{aligned}
$$

where $\Gamma_{M}(t):=\frac{\gamma\left(2^{-M} t\right)}{\gamma\left(2^{-M}\right)}, C_{M} \in[1,2)$ is a constant depending only on $M$.

By the fact that

$$
\left|\mathfrak{m}_{I, \gamma}^{j, k}\left(\widetilde{\xi}_{1}, \widetilde{\xi}_{2}\right)-\overline{\mathfrak{m}}_{I, \gamma}^{j, k}\left(\widetilde{\xi}_{1}, \widetilde{\xi}_{2}\right)\right| \leq \sum_{j, k \in \mathbb{Z}}\left|\mathfrak{m}_{I, \gamma}^{j, k}\left(\widetilde{\xi}_{1}, \widetilde{\xi}_{2}\right)-\overline{\mathfrak{m}}_{I, \gamma}^{j, k}\left(\widetilde{\xi}_{1}, \widetilde{\xi}_{2}\right)\right|
$$

it suffices to prove

$$
\sum_{j, k \in \mathbb{Z}}\left|\mathfrak{m}_{I, \gamma}^{j, k}\left(\widetilde{\xi}_{1}, \widetilde{\xi}_{2}\right)-\overline{\mathfrak{m}}_{I, \gamma}^{j, k}\left(\widetilde{\xi}_{1}, \widetilde{\xi}_{2}\right)\right| \lesssim 1
$$

for all $\left|\widetilde{\xi}_{1}\right|,\left|\widetilde{\xi}_{2}\right| \in[1,2)$, where the implicit constant is independent of $M$.

We now define the phase function of $\mathfrak{m}_{I, \gamma}^{j, k}$ by

$$
\varphi_{I, \gamma}^{j, k}(t):=2^{k} t \widetilde{\xi}_{1}+C_{M} 2^{j} \Gamma_{M}\left(2^{k} t\right) \widetilde{\xi}_{2}
$$

Then

$$
\left(\varphi_{I, \gamma}^{j, k}\right)^{\prime}(t)=2^{k} \widetilde{\xi}_{1}+C_{M} 2^{j} \Gamma_{M}^{\prime}\left(2^{k} t\right) 2^{k} \widetilde{\xi}_{2}
$$

and

$$
\left(\varphi_{I, \gamma}^{j, k}\right)^{\prime \prime}(t)=C_{M} 2^{j} \Gamma_{M}^{\prime \prime}\left(2^{k} t\right) 2^{2 k} \widetilde{\xi}_{2}
$$

To establish 2.6), we consider the following seven cases according to the values of $k$ and $j$. More precisely, for any given $k \geq 0$, we split $\mathbb{R}$ as a union of the following four intervals: $(-\infty, 1 / 8],\left(1 / 8, \Gamma_{M}^{\prime}\left(2^{k+1}\right)\right],\left(\Gamma_{M}^{\prime}\left(2^{k+1}\right), \frac{1}{8}\left(2 C_{1}\right)^{2 k} \Gamma_{M}^{\prime}(1)\right]$ and $\left(\frac{1}{8}\left(2 C_{1}\right)^{2 k} \Gamma_{M}^{\prime}(1), \infty\right)$, and divide 2.6 into four cases according to whether or not $\frac{1}{8} 2^{-j}$ belong to one of these intervals. For $k<0$, similarly, we will divide 2.6 into three cases.

Case 1: $k \geq 0$ and $\frac{1}{8} 2^{-j} \leq \frac{1}{8}$ (i.e., $j \geq 0$ ). From Theorem 1.2(ii), this implies that

$$
\left|\left(\varphi_{I, \gamma}^{j, k}\right)^{\prime \prime}(t)\right|=\left|C_{M} 2^{j} \frac{\Gamma_{M}^{\prime \prime}\left(2^{k} t\right) 2^{k} t}{\Gamma_{M}^{\prime}\left(2^{k} t\right)} \frac{\Gamma_{M}^{\prime}\left(2^{k} t\right)}{2^{k} t} 2^{2 k} \widetilde{\xi}_{2}\right| \gtrsim 2^{j} 2^{k} \Gamma_{M}^{\prime}\left(2^{k}\right) .
$$

By van der Corput's lemma, we obtain

$$
\left|\mathfrak{m}_{I, \gamma}^{j, k}\left(\widetilde{\xi}_{1}, \widetilde{\xi}_{2}\right)\right| \lesssim\left(2^{j} 2^{k} \Gamma_{M}^{\prime}\left(2^{k}\right)\right)^{-1 / 2}
$$


The same estimate also holds for $\overline{\mathfrak{m}}_{I, \gamma}^{j, k}\left(\widetilde{\xi}_{1}, \widetilde{\xi}_{2}\right)$, by 2.2 , we then have

$$
\sum_{\text {Case } 1}\left|\mathfrak{m}_{I, \gamma}^{j, k}\left(\widetilde{\xi}_{1}, \widetilde{\xi}_{2}\right)-\overline{\mathfrak{m}}_{I, \gamma}^{j, k}\left(\widetilde{\xi}_{1}, \widetilde{\xi}_{2}\right)\right| \lesssim \sum_{\text {Case } 1}\left(2^{j} 2^{k} \Gamma_{M}^{\prime}\left(2^{k}\right)\right)^{-1 / 2} \lesssim \sum_{\text {Case } 1}\left(2^{j} 2^{k}\right)^{-1 / 2} \lesssim 1
$$

for all $\left|\widetilde{\xi}_{1}\right|,\left|\widetilde{\xi}_{2}\right| \in[1,2)$.

Case 2: $k \geq 0$ and $\frac{1}{8}<\frac{1}{8} 2^{-j} \leq \Gamma_{M}^{\prime}\left(2^{k+1}\right)$. By Remark 1.4 and Theorem 1.2 (i), we have $-\log _{2}\left(8 C_{1} C_{1}^{\prime}\right)-k \log _{2} C_{1} \leq j<0$ and $2^{j} \Gamma_{M}^{\prime}\left(2^{k}\right) \geq \frac{1}{8 C_{1}}$. As in Case 1, it follows that

$$
\begin{aligned}
\sum_{\text {Case } 2}\left|\mathfrak{m}_{I, \gamma}^{j, k}\left(\widetilde{\xi}_{1}, \widetilde{\xi}_{2}\right)-\overline{\mathfrak{m}}_{I, \gamma}^{j, k}\left(\widetilde{\xi}_{1}, \widetilde{\xi}_{2}\right)\right| & \lesssim \sum_{\text {Case } 2}\left(2^{j} 2^{k} \Gamma_{M}^{\prime}\left(2^{k}\right)\right)^{-1 / 2} \lesssim \sum_{\text {Case } 2} 2^{-k / 2} \\
& \lesssim \sum_{k \geq 0}\left(\log _{2}\left(8 C_{1} C_{1}^{\prime}\right)+k \log _{2} C_{1}\right) 2^{-k / 2} \lesssim 1
\end{aligned}
$$

for all $\left|\widetilde{\xi}_{1}\right|,\left|\widetilde{\xi}_{2}\right| \in[1,2)$.

Case $3: k \geq 0$ and $\Gamma_{M}^{\prime}\left(2^{k+1}\right)<\frac{1}{8} 2^{-j} \leq \frac{1}{8}\left(2 C_{1}\right)^{2 k} \Gamma_{M}^{\prime}(1)$. From $\Gamma_{M}^{\prime}\left(2^{k+1}\right)<\frac{1}{8} 2^{-j}$ and (2.7), this implies that

$$
\left|\left(\varphi_{I, \gamma}^{j, k}\right)^{\prime}(t)\right| \geq 2^{k}\left(\left|\widetilde{\xi}_{1}\right|-C_{M} 2^{j}\left|\Gamma_{M}^{\prime}\left(2^{k} t\right) \widetilde{\xi}_{2}\right|\right) \geq 2^{k}
$$

This, by van der Corput's lemma and the fact that $\left(\varphi_{I, \gamma}^{j, k}\right)^{\prime}(t)$ is monotonic on $[1,2)$ or $(-2,-1]$, leads to

$$
\left|\mathfrak{m}_{I, \gamma}^{j, k}\left(\widetilde{\xi}_{1}, \widetilde{\xi}_{2}\right)\right| \lesssim 2^{-k}
$$

For $\overline{\mathfrak{m}}_{I, \gamma}^{j, k}\left(\widetilde{\xi}_{1}, \widetilde{\xi}_{2}\right)$, by van der Corput's lemma, it is easy to see that $\left|\int_{1 \leq|t|<2} e^{-i 2^{k} t \widetilde{\xi}_{1}} \mathrm{~d} t\right| \lesssim$ $2^{-k}$. This, combined with $\left|\int_{1 \leq|\tau|<2} e^{-i C_{M} 2^{j} \Gamma_{M}\left(2^{k} \tau\right) \widetilde{\xi}_{2}} \mathrm{~d} \tau\right| \lesssim 1$, further implies that

$$
\left|\overline{\mathfrak{m}}_{I, \gamma}^{j, k}\left(\widetilde{\xi}_{1}, \widetilde{\xi}_{2}\right)\right| \lesssim 2^{-k}
$$

Note that $|j| \lesssim k$, we have

$$
\sum_{\text {Case } 3}\left|\mathfrak{m}_{I, \gamma}^{j, k}\left(\widetilde{\xi}_{1}, \widetilde{\xi}_{2}\right)-\overline{\mathfrak{m}}_{I, \gamma}^{j, k}\left(\widetilde{\xi}_{1}, \widetilde{\xi}_{2}\right)\right| \lesssim \sum_{\text {Case } 3} 2^{-k} \lesssim \sum_{k \geq 0} k 2^{-k} \lesssim 1
$$

for all $\left|\widetilde{\xi}_{1}\right|,\left|\widetilde{\xi}_{2}\right| \in[1,2)$.

Case 4: $k \geq 0$ and $\frac{1}{8}\left(2 C_{1}\right)^{2 k} \Gamma_{M}^{\prime}(1)<\frac{1}{8} 2^{-j}$. The method of establishing 2.6) in this case is different from these cases above. Here, $\overline{\mathfrak{m}}_{I, \gamma}^{j, k}\left(\widetilde{\xi}_{1}, \widetilde{\xi}_{2}\right)$ plays a key role in this method, and we should consider $\left|\mathfrak{m}_{I, \gamma}^{j, k}\left(\widetilde{\xi}_{1}, \widetilde{\xi}_{2}\right)-\overline{\mathfrak{m}}_{I, \gamma}^{j, k}\left(\widetilde{\xi}_{1}, \widetilde{\xi}_{2}\right)\right|$, instead of considering $\left|\mathfrak{m}_{I, \gamma}^{j, k}\left(\widetilde{\xi}_{1}, \widetilde{\xi}_{2}\right)\right|$ and $\left|\overline{\mathfrak{m}}_{I, \gamma}^{j, k}\left(\widetilde{\xi}_{1}, \widetilde{\xi}_{2}\right)\right|$ separately. Indeed, we can rewrite $\mathfrak{m}_{I, \gamma}^{j, k}\left(\widetilde{\xi}_{1}, \widetilde{\xi}_{2}\right)-\overline{\mathfrak{m}}_{I, \gamma}^{j, k}\left(\widetilde{\xi}_{1}, \widetilde{\xi}_{2}\right)$ as

$$
\begin{gathered}
\int_{1 \leq|t|<2} e^{-i 2^{k} t \widetilde{\xi}_{1}}\left(e^{-i C_{M} 2^{j} \Gamma_{M}\left(2^{k} t\right) \widetilde{\xi}_{2}}-1\right) \mathrm{d} t \\
-\int_{1 \leq|t|<2} e^{-i 2^{k} t \widetilde{\xi}_{1}} \int_{1 \leq|\tau|<2}\left(e^{-i C_{M} 2^{j} \Gamma_{M}\left(2^{k} \tau\right) \widetilde{\xi}_{2}}-1\right) \mathrm{d} \tau \mathrm{d} t .
\end{gathered}
$$


By 2.2), Remark 1.4 and Theorem 1.2(i), we have

$$
\begin{aligned}
& \left|e^{-i C_{M} 2^{j} \Gamma_{M}\left(2^{k} t\right) \widetilde{\xi}_{2}}-1\right| \leq\left|C_{M} 2^{j} \Gamma_{M}\left(2^{k} t\right) \widetilde{\xi}_{2}\right| \leq \frac{8 C_{1}}{C_{2}+1} C_{1}^{\prime} 2^{j}\left(2 C_{1}\right)^{k}, \\
& \left|e^{-i C_{M} 2^{j} \Gamma_{M}\left(2^{k} \tau\right) \widetilde{\xi}_{2}}-1\right| \leq\left|C_{M} 2^{j} \Gamma_{M}\left(2^{k} \tau\right) \widetilde{\xi}_{2}\right| \leq \frac{8 C_{1}}{C_{2}+1} C_{1}^{\prime} 2^{j}\left(2 C_{1}\right)^{k} .
\end{aligned}
$$

Furthermore, an application of $\sum_{j: 2^{j} \lesssim\left(2 C_{1}\right)^{-2 k}} 2^{j} \lesssim\left(2 C_{1}\right)^{-2 k}$ then shows that

$$
\sum_{\text {Case } 4}\left|\mathfrak{m}_{I, \gamma}^{j, k}\left(\widetilde{\xi}_{1}, \widetilde{\xi}_{2}\right)-\overline{\mathfrak{m}}_{I, \gamma}^{j, k}\left(\widetilde{\xi}_{1}, \widetilde{\xi}_{2}\right)\right| \lesssim \sum_{\text {Case } 4} 2^{j}\left(2 C_{1}\right)^{k} \lesssim \sum_{k \geq 0}\left(2 C_{1}\right)^{-k} \lesssim 1
$$

for all $\left|\widetilde{\xi}_{1}\right|,\left|\widetilde{\xi}_{2}\right| \in[1,2)$.

Case 5: $k<0$ and $\left(2 e^{C_{2} / 2}\right)^{k / 2} \leq 2^{-j}$. As in Case 4, we may then apply (2.1), (2.2) and Remark 1.4 to conclude that

$$
\left|\mathfrak{m}_{I, \gamma}^{j, k}\left(\widetilde{\xi}_{1}, \widetilde{\xi}_{2}\right)-\overline{\mathfrak{m}}_{I, \gamma}^{j, k}\left(\widetilde{\xi}_{1}, \widetilde{\xi}_{2}\right)\right| \lesssim 2^{j}\left(2 e^{C_{2} / 2}\right)^{k}
$$

Therefore, by $\sum_{j: 2^{j} \lesssim\left(2 e^{C_{2} / 2}\right)^{-k / 2}} 2^{j} \lesssim\left(2 e^{C_{2} / 2}\right)^{-k / 2}$, it follows that

$$
\sum_{\text {Case } 5}\left|\mathfrak{m}_{I, \gamma}^{j, k}\left(\widetilde{\xi}_{1}, \widetilde{\xi}_{2}\right)-\overline{\mathfrak{m}}_{I, \gamma}^{j, k}\left(\widetilde{\xi}_{1}, \widetilde{\xi}_{2}\right)\right| \lesssim \sum_{\text {Case } 5} 2^{j}\left(2 e^{C_{2} / 2}\right)^{k} \lesssim \sum_{k<0}\left(2 e^{C_{2} / 2}\right)^{k / 2} \lesssim 1
$$

for all $\left|\widetilde{\xi}_{1}\right|,\left|\widetilde{\xi}_{2}\right| \in[1,2)$.

Case 6: $k<0$ and $\left(2 C_{1}\right)^{2 k}<2^{-j}<\left(2 e^{C_{2} / 2}\right)^{k / 2}$. In this case, we should rewrite $\mathfrak{m}_{I, \gamma}^{j, k}\left(\widetilde{\xi}_{1}, \widetilde{\xi}_{2}\right)-\overline{\mathfrak{m}}_{I, \gamma}^{j, k}\left(\widetilde{\xi}_{1}, \widetilde{\xi}_{2}\right)$ as

$$
\begin{gathered}
\int_{1 \leq|t|<2}\left(e^{-i 2^{k} t \widetilde{\xi}_{1}}-1\right) e^{-i C_{M} 2^{j} \Gamma_{M}\left(2^{k} t\right) \widetilde{\xi}_{2}} \mathrm{~d} t \\
-\int_{1 \leq|\tau|<2} e^{-i C_{M} 2^{j} \Gamma_{M}\left(2^{k} \tau\right) \widetilde{\xi}_{2}} \int_{1 \leq|t|<2}\left(e^{-i 2^{k} t \widetilde{\xi}_{1}}-1\right) \mathrm{d} t \mathrm{~d} \tau .
\end{gathered}
$$

From $\left|e^{-i 2^{k} t \widetilde{\xi}_{1}}-1\right| \leq\left|2^{k} t \widetilde{\xi}_{1}\right| \lesssim 2^{k}$, this implies $\left|\mathfrak{m}_{I, \gamma}^{j, k}\left(\widetilde{\xi}_{1}, \widetilde{\xi}_{2}\right)-\overline{\mathfrak{m}}_{I, \gamma}^{j, k}\left(\widetilde{\xi}_{1}, \widetilde{\xi}_{2}\right)\right| \lesssim 2^{k}$. Thus, noting the fact that $|j| \lesssim|k|$, we have

$$
\sum_{\text {Case } 6}\left|\mathfrak{m}_{I, \gamma}^{j, k}\left(\widetilde{\xi}_{1}, \widetilde{\xi}_{2}\right)-\overline{\mathfrak{m}}_{I, \gamma}^{j, k}\left(\widetilde{\xi}_{1}, \widetilde{\xi}_{2}\right)\right| \lesssim \sum_{\text {Case } 6} 2^{k} \lesssim \sum_{k<0}|k| 2^{k} \lesssim 1
$$

for all $\left|\widetilde{\xi}_{1}\right|,\left|\widetilde{\xi}_{2}\right| \in[1,2)$.

Case $7: k<0$ and $2^{-j} \leq\left(2 C_{1}\right)^{2 k}$. As in Case 1 , by $\sum_{j: 2^{-j} \leq\left(2 C_{1}\right)^{2 k}} 2^{-j / 2} \lesssim\left(2 C_{1}\right)^{k}$ and 2.2 , we have

$$
\begin{aligned}
\sum_{\text {Case } 7}\left|\mathfrak{m}_{I, \gamma}^{j, k}\left(\widetilde{\xi}_{1}, \widetilde{\xi}_{2}\right)-\overline{\mathfrak{m}}_{I, \gamma}^{j, k}\left(\widetilde{\xi}_{1}, \widetilde{\xi}_{2}\right)\right| & \lesssim \sum_{\text {Case } 7}\left(2^{j} 2^{k} \Gamma_{M}^{\prime}\left(2^{k}\right)\right)^{-1 / 2} \lesssim \sum_{\text {Case } 7}\left(2^{j}\left(2 C_{1}\right)^{k}\right)^{-1 / 2} \\
& \lesssim \sum_{k<0}\left(2 C_{1}\right)^{k / 2} \lesssim 1
\end{aligned}
$$

for all $\left|\widetilde{\xi}_{1}\right|,\left|\widetilde{\xi}_{2}\right| \in[1,2)$.

Altogether, we obtain 2.4 with $p=2$. 


\section{2. $L^{p}\left(\mathbb{R}^{2}\right)$-boundedness}

In Subsection 2.1. we have obtained the $L^{2}\left(\mathbb{R}^{2}\right)$-boundedness of $\sup _{j, k \in \mathbb{Z}}\left|\left(M_{I, \gamma}^{j, k}-\bar{M}_{I, \gamma}^{j, k}\right) f\right|$. Therefore, the $L^{p}\left(\mathbb{R}^{2}\right)$-boundedness, $p \in(2, \infty)$, of $\sup _{j, k \in \mathbb{Z}}\left|\left(M_{I, \gamma}^{j, k}-\bar{M}_{I, \gamma}^{j, k}\right) f\right|$ in 2.4 can be followed by interpolating with the trivial $L^{\infty}\left(\mathbb{R}^{2}\right)$-boundedness. The purpose of this subsection is to prove $(2.4)$ for all $p \in(1,2)$ in terms of the method of bootstrapping an iterated interpolation argument in the spirit of Nagel, Stein and Wainger in [23].

As in Carlsson, Christ, Córdoba et al. [7], we should first describe a Littlewood-Paley decomposition. Let us set

$$
\alpha_{k}:=\gamma^{\prime}\left(2^{k}\right) \text { and } \widehat{\mathbb{P}_{k} f}\left(\xi_{1}, \xi_{2}\right):=\Phi_{k}\left(\xi_{1}, \xi_{2}\right) \widehat{f}\left(\xi_{1}, \xi_{2}\right),
$$

where

$$
\operatorname{supp} \Phi_{k} \subseteq\left\{\left(\xi_{1}, \xi_{2}\right) \in \mathbb{R}^{2}: \alpha_{k-1} \leq\left|\xi_{1} / \xi_{2}\right| \leq \alpha_{k+1}\right\} .
$$

From [7], we also have

$$
\sum_{k \in \mathbb{Z}} \mathbb{P}_{k} f=f
$$

and

$$
\left\|\left(\sum_{k \in \mathbb{Z}}\left|\mathbb{P}_{k} f\right|^{2}\right)^{1 / 2}\right\|_{L^{p}\left(\mathbb{R}^{2}\right)} \lesssim\|f\|_{L^{p}\left(\mathbb{R}^{2}\right)}
$$

for all $p \in(1, \infty)$.

On the other hand, for any given $k \in \mathbb{Z}$, let us define a function $\psi_{k}(j)$ satisfying

$$
2^{j} \gamma^{\prime}\left(2^{k}\right)=\gamma^{\prime}\left(2^{k+\psi_{k}(j)}\right)
$$

It is easy to see that $\psi_{k}$ is monotonic and $\psi_{k}(0)=0$, which leads to the fact that there exists the inverse function $\left(\psi_{k}\right)^{-1}$ of $\psi_{k}$. From (2.8), we write

$$
\sup _{j, k \in \mathbb{Z}}\left|\left(M_{I, \gamma}^{j, k}-\bar{M}_{I, \gamma}^{j, k}\right) f\right|=\sup _{j, k \in \mathbb{Z}}\left|\left(M_{I, \gamma}^{j, k}-\bar{M}_{I, \gamma}^{j, k}\right)\left(\sum_{l \in \mathbb{Z}} \mathbb{P}_{l+k+\psi_{k}(j)} f\right)\right| .
$$

Therefore, for (2.4), by the triangle inequality, it is enough to prove that there exists a positive constant $\alpha$ such that

$$
\left\|\sup _{j, k \in \mathbb{Z}}\left|\left(M_{I, \gamma}^{j, k}-\bar{M}_{I, \gamma}^{j, k}\right)\left(\mathbb{P}_{l+k+\psi_{k}(j)} f\right)\right|\right\|_{L^{p}\left(\mathbb{R}^{2}\right)} \lesssim 2^{-\alpha|l|}\|f\|_{L^{p}\left(\mathbb{R}^{2}\right)}
$$

for all $p \in(1,2)$, which is equivalent to

$$
\left\|\sup _{j, k \in \mathbb{Z}}\left|\left(M_{I, \gamma}^{\left(\psi_{k}\right)^{-1}(j-l-k), k}-\bar{M}_{I, \gamma}^{\left(\psi_{k}\right)^{-1}(j-l-k), k}\right)\left(\mathbb{P}_{j} f\right)\right|\right\|_{L^{p}\left(\mathbb{R}^{2}\right)} \lesssim 2^{-\alpha|l|}\|f\|_{L^{p}\left(\mathbb{R}^{2}\right)} .
$$


Furthermore, by 2.9 , it suffices to obtain

$$
\begin{aligned}
& \left\|\left[\sum_{j \in \mathbb{Z}} \sup _{k \in \mathbb{Z}}\left|\left(M_{I, \gamma}^{\left(\psi_{k}\right)^{-1}(j-l-k), k}-\bar{M}_{I, \gamma}^{\left(\psi_{k}\right)^{-1}(j-l-k), k}\right)\left(\mathbb{P}_{j} f\right)\right|^{2}\right]^{1 / 2}\right\|_{L^{p}\left(\mathbb{R}^{2}\right)} \\
\lesssim & 2^{-\alpha|l|}\left\|\left(\sum_{j \in \mathbb{Z}}\left|\mathbb{P}_{j} f\right|^{2}\right)^{1 / 2}\right\| \|_{L^{p}\left(\mathbb{R}^{2}\right)}
\end{aligned}
$$

for all $p \in(1,2)$. By interpolation, we need to establish only the following estimates

$$
\begin{aligned}
&\left\|\left[\sum_{j \in \mathbb{Z}} \sup _{k \in \mathbb{Z}}\left|\left(M_{I, \gamma}^{\left(\psi_{k}\right)^{-1}(j-l-k), k}-\bar{M}_{I, \gamma}^{\left(\psi_{k}\right)^{-1}(j-l-k), k}\right)\left(\mathbb{P}_{j} f\right)\right|^{2}\right]^{1 / 2}\right\|_{L^{2}\left(\mathbb{R}^{2}\right)} \\
& \lesssim 2^{-\alpha|l|}\left\|\left(\sum_{j \in \mathbb{Z}}\left|\mathbb{P}_{j} f\right|^{2}\right)^{1 / 2}\right\|_{L^{2}\left(\mathbb{R}^{2}\right)}
\end{aligned}
$$

and

$$
\begin{aligned}
& \left\|\left[\sum_{j \in \mathbb{Z}} \sup _{k \in \mathbb{Z}}\left|\left(M_{I, \gamma}^{\left(\psi_{k}\right)^{-1}(j-l-k), k}-\bar{M}_{I, \gamma}^{\left(\psi_{k}\right)^{-1}(j-l-k), k}\right)\left(\mathbb{P}_{j} f\right)\right|^{2}\right]^{1 / 2}\right\|_{L^{p}\left(\mathbb{R}^{2}\right)} \\
\lesssim & \left\|\left(\sum_{j \in \mathbb{Z}}\left|\mathbb{P}_{j} f\right|^{2}\right)^{1 / 2}\right\|_{L^{p}\left(\mathbb{R}^{2}\right)}
\end{aligned}
$$

for all $p \in(1,2)$.

Proposition 2.1. We have

(1) 2.11 followed from

$$
\left\|\sup _{k \in \mathbb{Z}}\left|\left(M_{I, \gamma}^{\left(\psi_{k}\right)^{-1}(j-l-k), k}-\bar{M}_{I, \gamma}^{\left(\psi_{k}\right)^{-1}(j-l-k), k}\right)\left(\mathbb{P}_{j} f\right)\right|\right\|_{L^{2}\left(\mathbb{R}^{2}\right)} \lesssim 2^{-\alpha|l|}\left\|\mathbb{P}_{j} f\right\|_{L^{2}\left(\mathbb{R}^{2}\right)}
$$

(2) 2.12 followed from

$$
\left\|\sup _{k \in \mathbb{Z}}\left|\left(M_{I, \gamma}^{\left(\psi_{k}\right)^{-1}(j-l-k), k}-\bar{M}_{I, \gamma}^{\left(\psi_{k}\right)^{-1}(j-l-k), k}\right)\left(\mathbb{P}_{j} f\right)\right|\right\|_{L^{p}\left(\mathbb{R}^{2}\right)} \lesssim\left\|\mathbb{P}_{j} f\right\|_{L^{p}\left(\mathbb{R}^{2}\right)}
$$

for all $p \in(1, \infty)$.

Proof. For 2.13 $\Rightarrow 2.11$, which comes from the commutative property between $l^{2}(\mathbb{Z})$ and $L^{2}\left(\mathbb{R}^{2}\right)$ norms. For $2.14 \Rightarrow 2.12$, we first consider the more general estimate

$$
\begin{aligned}
& \left\|\left[\sum_{j \in \mathbb{Z}} \sup _{k \in \mathbb{Z}}\left|\left(M_{I, \gamma}^{\left(\psi_{k}\right)^{-1}(j-l-k), k}-\bar{M}_{I, \gamma}^{\left(\psi_{k}\right)^{-1}(j-l-k), k}\right)\left(\mathbb{P}_{j} f\right)\right|^{q_{1}}\right]^{1 / q_{1}}\right\|_{L^{q_{2}\left(\mathbb{R}^{2}\right)}} \\
\lesssim & \left\|\left(\sum_{j \in \mathbb{Z}}\left|\mathbb{P}_{j} f\right|^{q_{1}}\right)^{1 / q_{1}}\right\|_{L^{q_{2}\left(\mathbb{R}^{2}\right)}}
\end{aligned}
$$


for certain $q_{1} \in(1, \infty]$ and $q_{2} \in(1, \infty)$.

For the case of $q_{1}=\infty$ and $q_{2}=2$, by $(2.9)$, it follows that

$$
\left\|\sup _{j \in \mathbb{Z}}\left|\mathbb{P}_{j} f\right|\right\|_{L^{p}\left(\mathbb{R}^{2}\right)} \leq\left\|\left(\sum_{l \in \mathbb{Z}}\left|\mathbb{P}_{j} f\right|^{2}\right)^{1 / 2}\right\|_{L^{p}\left(\mathbb{R}^{2}\right)} \lesssim\|f\|_{L^{p}\left(\mathbb{R}^{2}\right)}
$$

for all $p \in(1, \infty)$. Recall that in Subsection 2.1, we have obtained

$$
\left\|\sup _{j, k \in \mathbb{Z}} M_{I, \gamma}^{\left(\psi_{k}\right)^{-1}(j-l-k), k} f\right\|_{L^{2}\left(\mathbb{R}^{2}\right)} \lesssim\|f\|_{L^{2}\left(\mathbb{R}^{2}\right)}
$$

for all $f \in L^{2}\left(\mathbb{R}^{2}\right)$. Therefore,

$$
\begin{aligned}
\left\|\sup _{j, k \in \mathbb{Z}} M_{I, \gamma}^{\left(\psi_{k}\right)^{-1}(j-l-k), k}\left(\mathbb{P}_{j} f\right)\right\|_{L^{2}\left(\mathbb{R}^{2}\right)} & \lesssim\left\|\sup _{j, k \in \mathbb{Z}} M_{I, \gamma}^{\left(\psi_{k}\right)^{-1}(j-l-k), k}\left(\sup _{j \in \mathbb{Z}}\left|\mathbb{P}_{j} f\right|\right)\right\|_{L^{2}\left(\mathbb{R}^{2}\right)} \\
& \lesssim\left\|\sup _{j \in \mathbb{Z}}\left|\mathbb{P}_{j} f\right|\right\|_{L^{2}\left(\mathbb{R}^{2}\right)} \cdot
\end{aligned}
$$

From (2.3), we also have

$$
\begin{aligned}
\left\|\sup _{j, k \in \mathbb{Z}} \bar{M}_{I, \gamma}^{\left(\psi_{k}\right)^{-1}(j-l-k), k}\left(\mathbb{P}_{j} f\right)\right\|_{L^{2}\left(\mathbb{R}^{2}\right)} & \lesssim\left\|M^{(1)} M^{(2)}\left(\sup _{j \in \mathbb{Z}}\left|\mathbb{P}_{j} f\right|\right)\right\|_{L^{2}\left(\mathbb{R}^{2}\right)} \\
& \lesssim\left\|\sup _{j \in \mathbb{Z}}\left|\mathbb{P}_{j} f\right|\right\|_{L^{2}\left(\mathbb{R}^{2}\right)} .
\end{aligned}
$$

Thus, from (2.16) and (2.17), we obtain (2.15) in the case of $q_{1}=\infty$ and $q_{2}=2$.

For the case of $q_{1}=q_{2}$ and $q_{1}, q_{2} \in(1, \infty)$, because the $l^{p}(\mathbb{Z})$ and $L^{p}\left(\mathbb{R}^{2}\right)$ norms commute, we have that 2.15 is equivalent to 2.14 . Then, by the interpolation argument, we may obtain that (2.12) holds for all $p \in(4 / 3,2)$. Repeating the interpolation argument and using $q_{1}=\infty$ and $q_{2} \in(4 / 3,2)$, we may also obtain that 2.12 holds for all $p \in$ $(8 / 7,2)$. Reiterating this process sufficiently many times we can show that 2.12 holds for all $p \in(1,2)$. Hence, we obtain 2.14$) \Rightarrow 2.12$.

Therefore, it is enough to prove (2.13) and (2.14). Indeed, since the $L^{p}\left(\mathbb{R}^{2}\right)$-boundedness of $\sup _{j, k \in \mathbb{Z}}\left|\left(M_{I, \gamma}^{j, k}-\bar{M}_{I, \gamma}^{j, k}\right) f\right|$ has been obtained for all $p \in[2, \infty]$, we can restrict $p \in(1,2)$ in (2.14). We first bound the left-hand side of 2.14 by

$$
\left\|\left[\sum_{k \in \mathbb{Z}}\left|\left(M_{I, \gamma}^{\left(\psi_{k}\right)^{-1}(j-l-k), k}-\bar{M}_{I, \gamma}^{\left(\psi_{k}\right)^{-1}(j-l-k), k}\right)\left(\mathbb{P}_{j} f\right)\right|^{2}\right]^{1 / 2}\right\|_{L^{p}\left(\mathbb{R}^{2}\right)},
$$

and define a finer frequency decomposition operator $\mathbb{P}_{j,-k+m}$ by restricting the frequency region to

$$
\left\{\left(\xi_{1}, \xi_{2}\right) \in \mathbb{R}^{2}: 2^{-k+m-1} \leq\left|\xi_{1}\right|, \gamma^{\prime}\left(2^{j}\right)\left|\xi_{2}\right| \leq 2^{-k+m+1}\right\}
$$


Furthermore, we can write

$$
\mathbb{P}_{j} f=\sum_{m \in \mathbb{Z}} \mathbb{P}_{j,-k+m} f .
$$

Thus, for (2.14), by the triangle inequality, it suffices to prove that there exists a positive constant $\beta$ such that

$$
\begin{aligned}
&\left\|\left[\sum_{k \in \mathbb{Z}}\left|\left(M_{I, \gamma}^{\left(\psi_{k}\right)^{-1}(j-l-k), k}-\bar{M}_{I, \gamma}^{\left(\psi_{k}\right)^{-1}(j-l-k), k}\right)\left(\mathbb{P}_{j,-k+m} f\right)\right|^{2}\right]^{1 / 2}\right\|_{L^{p}\left(\mathbb{R}^{2}\right)} \\
& \lesssim 2^{-\beta|m|}\left\|\mathbb{P}_{j} f\right\|_{L^{p}\left(\mathbb{R}^{2}\right)}
\end{aligned}
$$

for all $p \in(1,2)$. As a result, we obtain $2.18 \Rightarrow 2.14$.

Proposition 2.2. We have that 2.13) and 2.18) can be followed from

$$
\begin{aligned}
& \left\|\left[\sum_{k \in \mathbb{Z}}\left|\left(M_{I, \gamma}^{\left(\psi_{k}\right)^{-1}(j-l-k), k}-\bar{M}_{I, \gamma}^{\left(\psi_{k}\right)^{-1}(j-l-k), k}\right)\left(\mathbb{P}_{j,-k+m} f\right)\right|^{2}\right]^{1 / 2}\right\|_{L^{2}\left(\mathbb{R}^{2}\right)} \\
\lesssim & 2^{-\alpha|l|} 2^{-\beta|m|}\left\|\mathbb{P}_{j} f\right\|_{L^{2}\left(\mathbb{R}^{2}\right)}
\end{aligned}
$$

for some positive constants $\alpha$ and $\beta$.

Proof. For 2.19$) \Rightarrow 2.13$, which can be proved as $2.18 \Rightarrow 2.14$. For 2.19$) \Rightarrow 2.18$, by interpolation and the Littlewood-Paley theory, it suffices to obtain

$$
\begin{aligned}
& \left\|\left[\sum_{k \in \mathbb{Z}}\left|\left(M_{I, \gamma}^{\left(\psi_{k}\right)^{-1}(j-l-k), k}-\bar{M}_{I, \gamma}^{\left(\psi_{k}\right)^{-1}(j-l-k), k}\right)\left(\mathbb{P}_{j,-k+m} f\right)\right|^{2}\right]^{1 / 2}\right\|_{L^{p}\left(\mathbb{R}^{2}\right)} \\
\lesssim & \left\|\left(\sum_{k \in \mathbb{Z}}\left|\mathbb{P}_{j,-k+m} f\right|^{2}\right)^{1 / 2}\right\| \|_{L^{p}\left(\mathbb{R}^{2}\right)}
\end{aligned}
$$

for all $p \in(1,2)$. Similarly, we consider the more general estimate

$$
\begin{aligned}
& \left\|\left[\sum_{k \in \mathbb{Z}}\left|\left(M_{I, \gamma}^{\left(\psi_{k}\right)^{-1}(j-l-k), k}-\bar{M}_{I, \gamma}^{\left(\psi_{k}\right)^{-1}(j-l-k), k}\right)\left(\mathbb{P}_{j,-k+m} f\right)\right|^{q_{1}}\right]^{1 / q_{1}}\right\|_{L^{q_{2}\left(\mathbb{R}^{2}\right)}} \\
\lesssim & \left\|\left(\sum_{k \in \mathbb{Z}}\left|\mathbb{P}_{j,-k+m} f\right|^{q_{1}}\right)^{1 / q_{1}}\right\| \|_{L^{q_{2}\left(\mathbb{R}^{2}\right)}}
\end{aligned}
$$

for certain $q_{1} \in(1, \infty]$ and $q_{2} \in(1, \infty)$.

As in 2.16) and (2.17), we have that 2.21) can be established for the case of $q_{1}=\infty$ and $q_{2}=2$. On the other hand, for the case of $q_{1}=q_{2}$ and $q_{1}, q_{2} \in(1, \infty)$, it is easy to see that 2.21 is equivalent to

$$
\left\|\left(M_{I, \gamma}^{\left(\psi_{k}\right)^{-1}(j-l-k), k}-\bar{M}_{I, \gamma}^{\left(\psi_{k}\right)^{-1}(j-l-k), k}\right)\left(\mathbb{P}_{j,-k+m} f\right)\right\|_{L^{q_{2}\left(\mathbb{R}^{2}\right)}} \lesssim\left\|\mathbb{P}_{j,-k+m} f\right\|_{L^{q_{2}\left(\mathbb{R}^{2}\right)}}
$$

for all $q_{2} \in(1, \infty)$, which is a trivial consequence of Minkowski's inequality. Therefore, as in 2.12 , we obtain 2.20 , which leads to $2.19 \Rightarrow 2.18$. 
In conclusion, we will only prove 2.19). For this aim, it is sufficient to show the following estimate

$$
\left\|\left(M_{I, \gamma}^{\left(\psi_{k}\right)^{-1}(j-l-k), k}-\bar{M}_{I, \gamma}^{\left(\psi_{k}\right)^{-1}(j-l-k), k}\right)\left(\mathbb{P}_{j,-k+m} f\right)\right\|_{L^{2}\left(\mathbb{R}^{2}\right)} \lesssim 2^{-\alpha|l|} 2^{-\beta|m|}\|f\|_{L^{2}\left(\mathbb{R}^{2}\right)} .
$$

This, by Plancherel's theorem, can be followed from

$$
\left|m_{I, \gamma}^{\left(\psi_{k}\right)^{-1}(j-l-k), k}\left(\xi_{1}, \xi_{2}\right)-\bar{m}_{I, \gamma}^{\left(\psi_{k}\right)^{-1}(j-l-k), k}\left(\xi_{1}, \xi_{2}\right)\right| \lesssim 2^{-\alpha|l|} 2^{-\beta|m|}
$$

for all $\left(\xi_{1}, \xi_{2}\right) \in \mathbb{R}^{2}$ satisfying

$$
\alpha_{j-1} \leq\left|\xi_{1} / \xi_{2}\right| \leq \alpha_{j+1} \quad \text { and } \quad 2^{-k+m-1} \leq\left|\xi_{1}\right|, \quad \gamma^{\prime}\left(2^{j}\right)\left|\xi_{2}\right| \leq 2^{-k+m+1} .
$$

On the other hand, we may assume that $|l|$ and $|m|$ large enough, since we have the following trivial estimates

$$
\left|m_{I, \gamma}^{\left(\psi_{k}\right)^{-1}(j-l-k), k}\left(\xi_{1}, \xi_{2}\right)\right| \lesssim 1 \quad \text { and } \quad\left|\bar{m}_{I, \gamma}^{\left(\psi_{k}\right)^{-1}(j-l-k), k}\left(\xi_{1}, \xi_{2}\right)\right| \lesssim 1
$$

for all $\left(\xi_{1}, \xi_{2}\right) \in \mathbb{R}^{2}$.

To prove 2.22), we consider the following five cases according to the values of $m$ and $l$.

Case I: $m \geq 0$ and $l \leq \frac{m}{2 \log _{2} C_{1}}$. From Theorem 1.2 (ii) and 2.10, this implies that

$$
\left|\left(\varphi_{I, \gamma}^{\left(\psi_{k}\right)^{-1}(j-l-k), k}\right)^{\prime \prime}(t)\right|=\left|2^{\left(\psi_{k}\right)^{-1}(j-l-k)} \frac{\gamma^{\prime \prime}\left(2^{k} t\right) 2^{k} t}{\gamma^{\prime}\left(2^{k} t\right)} \frac{\gamma^{\prime}\left(2^{k} t\right)}{2^{k} t} 2^{2 k} \xi_{2}\right| \gtrsim 2^{m} \frac{\gamma^{\prime}\left(2^{j-l}\right)}{\gamma^{\prime}\left(2^{j}\right)} .
$$

By Theorem 1.2(i) and (2.1), we obtain

$$
\begin{cases}\left(C_{1}\right)^{-l} \leq \frac{\gamma^{\prime}\left(2^{j-l}\right)}{\gamma^{\prime}\left(2^{j}\right)} \leq e^{-\frac{C_{2}}{2} l} & \text { for } l>0 \\ e^{-\frac{C_{2}}{2} l} \leq \frac{\gamma^{\prime}\left(2^{j-l}\right)}{\gamma^{\prime}\left(2^{j}\right)} \leq\left(C_{1}\right)^{-l} & \text { for } l<0\end{cases}
$$

which leads to

$$
\left|\left(\varphi_{I, \gamma}^{j, k}\right)^{\prime \prime}(t)\right| \gtrsim \begin{cases}2^{m}\left(C_{1}\right)^{-l} & \text { for } l>0 \\ 2^{m} e^{-\frac{C_{2}}{2} l} & \text { for } l<0\end{cases}
$$

By van der Corput's lemma, we obtain

$$
\left|m_{I, \gamma}^{\left(\psi_{k}\right)^{-1}(j-l-k), k}\left(\xi_{1}, \xi_{2}\right)\right| \lesssim \begin{cases}2^{-m / 2}\left(C_{1}\right)^{l / 2} & \text { for } l>0 \\ 2^{-m / 2} e^{\frac{C_{2}}{4} l} & \text { for } l<0 .\end{cases}
$$

This boundedness can also be obtained for $\bar{m}_{I, \gamma}^{\left(\psi_{k}\right)^{-1}(j-l-k), k}\left(\xi_{1}, \xi_{2}\right)$. Therefore,

$$
\left|m_{I, \gamma}^{\left(\psi_{k}\right)^{-1}(j-l-k), k}\left(\xi_{1}, \xi_{2}\right)-\bar{m}_{I, \gamma}^{\left(\psi_{k}\right)^{-1}(j-l-k), k}\left(\xi_{1}, \xi_{2}\right)\right| \lesssim \begin{cases}2^{-m / 2}\left(C_{1}\right)^{l / 2} & \text { for } l>0, \\ 2^{-m / 2} e^{\frac{C_{2}}{4} l} & \text { for } l<0 .\end{cases}
$$

To obtain 2.22 in this case, two different situations can arise. 
$\diamond$ For $l>0$, since $2^{-m / 2}\left(C_{1}\right)^{l / 2}=2^{-m / 8} 2^{-3 m / 8}\left(C_{1}\right)^{l / 2} \leq 2^{-m / 8}\left(C_{1}\right)^{-1 / 4}$, we have 2.22 established with $\alpha:=\frac{1}{4} \log _{2} C_{1}$ and $\beta:=1 / 8$.

$\diamond$ For $l<0$, we have 2.22 established with $\alpha:=\frac{C_{2}}{4} \log _{2} e$ and $\beta:=1 / 2$.

Case II: $m \geq 0$ and $\frac{m}{2 \log _{2} C_{1}}<l<\frac{4 m}{C_{2} \log _{2} e}$. From Theorem 1.2 (i) and 2.10 , we may conclude that

$$
\left|\left(\varphi_{I, \gamma}^{\left(\psi_{k}\right)^{-1}(j-l-k), k}\right)^{\prime}(t)\right| \geq 2^{k}\left|\xi_{1}\right|-\left|2^{\left(\psi_{k}\right)^{-1}(j-l-k)} \gamma^{\prime}\left(2^{k} t\right) 2^{k} \xi_{2}\right| \geq 2^{m}\left(\frac{1}{2}-2 C_{1} e^{-\frac{C_{2}}{2} l}\right) \gtrsim 2^{m}
$$

for $l$ and $m$ large enough. Notice the fact that $\left(\varphi_{I, \gamma}^{\left(\psi_{k}\right)^{-1}(j-l-k), k}\right)^{\prime}(t)$ is monotonic on $[1,2)$ or $(-2,-1]$, by van der Corput's lemma, we may obtain

$$
\left|m_{I, \gamma}^{\left(\psi_{k}\right)^{-1}(j-l-k), k}\left(\xi_{1}, \xi_{2}\right)\right| \lesssim 2^{-m}
$$

On the other hand, we also have that $\left|\bar{m}_{I, \gamma}^{\left(\psi_{k}\right)^{-1}(j-l-k), k}\left(\xi_{1}, \xi_{2}\right)\right|$ can be bounded by $2^{-m}$. Therefore,

$$
\left|m_{I, \gamma}^{\left(\psi_{k}\right)^{-1}(j-l-k), k}\left(\xi_{1}, \xi_{2}\right)-\bar{m}_{I, \gamma}^{\left(\psi_{k}\right)^{-1}(j-l-k), k}\left(\xi_{1}, \xi_{2}\right)\right| \lesssim 2^{-m} \leq 2^{-m / 2} 2^{-\frac{C_{2} \log _{2} e}{8} l},
$$

which proves 2.22 with $\alpha:=\frac{C_{2} \log _{2} e}{8}$ and $\beta:=1 / 2$.

Case III: $m \geq 0$ and $l \geq \frac{4 m}{C_{2} \log _{2} e}$, or $m<0$ and $\frac{m}{2 \log _{2} C_{1}} \leq l$. As in Case 4 in Subsection 2.1, by 2.2 and Theorem 1.2(i), we see that

$\left|m_{I, \gamma}^{\left(\psi_{k}\right)^{-1}(j-l-k), k}\left(\xi_{1}, \xi_{2}\right)-\bar{m}_{I, \gamma}^{\left(\psi_{k}\right)^{-1}(j-l-k), k}\left(\xi_{1}, \xi_{2}\right)\right| \lesssim 2^{m} \frac{\gamma^{\prime}\left(2^{j-l}\right)}{\gamma^{\prime}\left(2^{j}\right)} \lesssim \begin{cases}2^{m} e^{-\frac{C_{2}}{2} l} & \text { for } l>0 \\ 2^{m}\left(C_{1}\right)^{-l} & \text { for } l<0 .\end{cases}$

For obtaining 2.22 in this case, we consider the following three cases.

$\diamond$ For the case of $m \geq 0$ and $l \geq \frac{4 m}{C_{2} \log _{2} e}$, note that $2^{m} e^{-\frac{C_{2}}{2} l}=2^{m} e^{-\frac{3 C_{2}}{8} l} e^{-\frac{C_{2}}{8} l} \leq$ $2^{-m / 2} e^{-\frac{C_{2}}{8} l}$, we then establish 2.22 with $\alpha:=\frac{C_{2} \log _{2} e}{8}$ and $\beta:=1 / 2$.

$\diamond$ For the case of $m<0$ and $l>0$, it is easy to see that 2.22 established with $\alpha:=\frac{C_{2} \log _{2} e}{2}$ and $\beta:=1$.

$\diamond$ For the case of $m<0$ and $\frac{m}{2 \log _{2} C_{1}} \leq l \leq 0$, we may take $|l|$ and $|m|$ large enough. Noting that $2^{m}\left(C_{1}\right)^{-l}=2^{\frac{m}{4}} 2^{\frac{3 m}{4}}\left(C_{1}\right)^{-l} \leq 2^{\frac{m}{4}}\left(C_{1}\right)^{l / 2}$, we then establish 2.22 with $\alpha:=\frac{\log _{2} C_{1}}{2}$ and $\beta:=1 / 4$.

Case IV: $m<0$ and $\frac{4 m}{C_{2} \log _{2} e} \leq l \leq \frac{m}{2 \log _{2} C_{1}}$. As in Case 6 in Subsection 2.1, we have

$$
\left|m_{I, \gamma}^{\left(\psi_{k}\right)^{-1}(j-l-k), k}\left(\xi_{1}, \xi_{2}\right)-\bar{m}_{I, \gamma}^{\left(\psi_{k}\right)^{-1}(j-l-k), k}\left(\xi_{1}, \xi_{2}\right)\right| \lesssim 2^{m} \leq 2^{m / 2} e^{\frac{C_{2}}{8} l} .
$$


We then establish 2.22 with $\alpha:=\frac{C_{2} \log _{2} e}{8}$ and $\beta:=1 / 2$.

Case V: $m<0$ and $l<\frac{4 m}{C_{2} \log _{2} e}$. From Theorem 1.2(i) and 2.10, this implies that

$$
\begin{aligned}
\left|\left(\varphi_{I, \gamma}^{\left(\psi_{k}\right)^{-1}(j-l-k), k}\right)^{\prime}(t)\right| & \geq\left|2^{\left(\psi_{k}\right)^{-1}(j-l-k)} \gamma^{\prime}\left(2^{k} t\right) 2^{k} \xi_{2}\right|-2^{k}\left|\xi_{1}\right| \\
& \geq 2^{m}\left(\frac{e^{-\frac{C_{2}}{2} l}}{2}-2\right) \gtrsim 2^{m} e^{-\frac{C_{2}}{2} l}
\end{aligned}
$$

for $|l|$ and $|m|$ large enough. We notice that $\left(\varphi_{I, \gamma}^{\left(\psi_{k}\right)^{-1}(j-l-k), k}\right)^{\prime}(t)$ is monotonic on $[1,2)$ or $(-2,-1]$, van der Corput's lemma yields

$$
\left|m_{I, \gamma}^{\left(\psi_{k}\right)^{-1}(j-l-k), k}\left(\xi_{1}, \xi_{2}\right)\right| \lesssim 2^{-m} e^{\frac{C_{2}}{2} l} .
$$

On the other hand, we also have $\left|\bar{m}_{I, \gamma}^{\left(\psi_{k}\right)^{-1}(j-l-k), k}\left(\xi_{1}, \xi_{2}\right)\right|$ can be bounded by $2^{-m} e^{\frac{C_{2}}{2} l}$, which further leads to

$$
\left|m_{I, \gamma}^{\left(\psi_{k}\right)^{-1}(j-l-k), k}\left(\xi_{1}, \xi_{2}\right)-\bar{m}_{I, \gamma}^{\left(\psi_{k}\right)^{-1}(j-l-k), k}\left(\xi_{1}, \xi_{2}\right)\right| \lesssim 2^{-m} e^{\frac{C_{2}}{2} l} \leq 2^{m / 2} e^{\frac{C_{2}}{8} l} .
$$

We then establish $(2.22)$ with $\alpha:=\frac{C_{2} \log _{2} e}{8}$ and $\beta:=1 / 2$.

In total, we obtain 2.22 with $\alpha:=\frac{C_{2} \log _{2} e}{8}$ and $\beta:=1 / 8$. This finishes the proof of Theorem 1.2 ,

\section{Proof of Theorem 1.3}

For $M_{I I, \gamma}$, the method applied in $M_{I, \gamma}$ is no longer available, and therefore we must find a different method to prove Theorem 1.3 . We first restrict $p \in(1, \infty)$ based on the trivial $L^{\infty}\left(\mathbb{R}^{2}\right)$-boundedness of $M_{I I, \gamma}$, and also introduce some simple properties of the curve $\gamma$. Indeed, from Theorem 1.3(i) and (2.2), we have

$$
C_{2}+1 \leq \frac{t \gamma^{\prime}(t)}{\gamma(t)} \leq C_{1}^{\prime} \quad \text { for any } t \in(0, \infty) .
$$

As in 2.1), if consider $F(t):=\ln \gamma(t)$ for any $t \in(0, \infty)$, by (3.1), we have

$$
e^{\left(C_{2}+1\right) / 2} \leq \frac{\gamma(2 t)}{\gamma(t)} \leq e^{C_{1}^{\prime}} \quad \text { for any } t \in(0, \infty)
$$

As in $M_{I, \gamma}$, we may assume that $f$ is non-negative. Let $\phi: \mathbb{R} \rightarrow \mathbb{R}$ be a smooth function supported on $\{t \in \mathbb{R}: 1 / 2 \leq|t| \leq 2\}$ with the properties that $0 \leq \phi(t) \leq 1$ and $\sum_{l \in \mathbb{Z}} \phi_{l}(t)=1$ for any $t \neq 0$, where $\phi_{l}(t):=\phi\left(2^{-l} t\right)$. Here and hereafter, for any $l \in \mathbb{Z}$, we denote $P_{l}^{(1)}$ and $P_{l}^{(2)}$ as the Littlewood-Paley projection corresponding to $\phi_{l}$ in the first variable and the second variable, respectively,

$$
\begin{aligned}
& P_{l}^{(1)} f\left(x_{1}, x_{2}\right):=\int_{-\infty}^{\infty} f\left(x_{1}-w, x_{2}\right) \check{\phi}_{l}(w) \mathrm{d} w \\
& P_{l}^{(2)} f\left(x_{1}, x_{2}\right):=\int_{-\infty}^{\infty} f\left(x_{1}, x_{2}-w\right) \check{\phi}_{l}(w) \mathrm{d} w .
\end{aligned}
$$


For any given $l \in \mathbb{Z}$, set

$$
M_{I I, l}^{i, j} f\left(x_{1}, x_{2}\right):=\int_{-\infty}^{\infty} f\left(x_{1}-2^{i} t, x_{2}-2^{j} \gamma(t)\right) \phi_{l}(t) \frac{\mathrm{d} t}{|t|} .
$$

Then, as in $M_{I, \gamma}$, it suffices to prove the $L^{p}\left(\mathbb{R}^{2}\right)$-boundedness of the operator $\sup _{i, j, l \in \mathbb{Z}} M_{I I, l}^{i, j}$. Furthermore, for simplicity, we let $z:=\left(x_{1}, x_{2}\right), i_{z}:=i\left(x_{1}, x_{2}\right), j_{z}:=j\left(x_{1}, x_{2}\right)$ and $l_{z}:=l\left(x_{1}, x_{2}\right)$ be measurable functions. By linearization, it suffices to prove the $L^{p}\left(\mathbb{R}^{2}\right)$ boundedness of the operator $M_{I I, l_{z}}^{i_{z}, j_{z}}$.

Note that $\gamma$ is strictly increasing on $\mathbb{R}^{+}$with $\gamma\left(\mathbb{R}^{+}\right)=\mathbb{R}^{+}$. For these $j_{z}, l_{z} \in \mathbb{Z}$, we denote by $k_{z}: \mathbb{R}^{2} \rightarrow \mathbb{R}$ the measurable function satisfying

$$
2^{k_{z}} 2^{j_{z}} \gamma\left(2^{l_{z}}\right)=1
$$

We then write $M_{I I, l_{z}}^{i_{z}, j_{z}} f=\sum_{k \in \mathbb{Z}} M_{I I, l_{z}}^{i_{z}, j_{z}} P_{k}^{(2)} f$, and further split it as the sum of the following low-frequency part $M_{I I_{a}, l_{z}}^{i_{z}, j_{z}} f$ and the high-frequency part $M_{I I_{b}, l_{z}}^{i_{z}, j_{z}} f$, where

$$
M_{I I_{a}, l_{z}}^{i_{z}, j_{z}} f:=\sum_{k \leq k_{z}} M_{I I, l_{z}}^{i_{z}, j_{z}} P_{k}^{(2)} f \quad \text { and } \quad M_{I I_{b}, l_{z}}^{i_{z}, j_{z}} f:=\sum_{k>k_{z}} M_{I I, l_{z}}^{i_{z}, j_{z}} P_{k}^{(2)} f .
$$

Consider $M_{I I_{a}, l_{z}}^{i_{z}, j_{z}} f$. We compare it with the following operator

$$
\mathbb{M}_{I I_{a}, l_{z}}^{i_{z}, j_{z}} f\left(x_{1}, x_{2}\right):=\sum_{k \leq k_{z}} \int_{-\infty}^{\infty} P_{k}^{(2)} f\left(x_{1}-2^{i_{z}} t, x_{2}\right) \phi_{l_{z}}(t) \frac{\mathrm{d} t}{|t|}
$$

It is easy to see that $\left|\mathbb{M}_{I I_{a}, l_{z}}^{i_{z}, j_{z}} f\right|$ can be bounded by $M^{(1)}\left(\sum_{k \leq k_{z}} P_{k}^{(2)} f\right)$. Note that the operator $\sum_{k \leq k_{z}} P_{k}^{(2)} f$ is bounded on $L^{p}\left(\mathbb{R}^{2}\right)$ for any given $p \in(1, \infty)$ by multiplier theory, then we can use the $L^{p}\left(\mathbb{R}^{2}\right)$-boundedness of $M^{(1)}$ to get

$$
\left\|\mathbb{M}_{I I_{a}, l_{z}}^{i_{z}, j_{z}} f\right\|_{L^{p}\left(\mathbb{R}^{2}\right)} \lesssim\|f\|_{L^{p}\left(\mathbb{R}^{2}\right)}
$$

for all $p \in(1, \infty)$. The difference between $M_{I I_{a}, l_{z}}^{i_{z}, j_{z}} f(z)$ and $\mathbb{M}_{I I_{a}, l_{z}}^{i_{z}, j_{z}} f(z)$ can be written as

$$
\sum_{k \leq k_{z}} \int_{-\infty}^{\infty} \int_{-\infty}^{\infty} f\left(x_{1}-2^{i_{z}} t, x_{2}-w\right)\left(\check{\phi}_{k}\left(w-2^{j_{z}} \gamma(t)\right)-\check{\phi}_{k}(w)\right) \mathrm{d} w \phi_{l z}(t) \frac{\mathrm{d} t}{|t|} .
$$

By the Lagrange mean value theorem, we have

$$
\left|\check{\phi}_{k}\left(w-2^{j_{z}} \gamma(t)\right)-\check{\phi}_{k}(w)\right| \lesssim 2^{j_{z}}|\gamma(t)| 2^{2 k} 2^{-2 n}
$$

if $2^{j_{z}}|\gamma(t)| \leq 2^{-k}$ and $w$ is in the annulus $2^{-k+n-1} \leq|w| \leq 2^{-k+n}$ for $n \in \mathbb{N}$. For $n=0$ the above estimate holds for all $|w| \leq 2^{-k}$. For $t \in \operatorname{supp} \psi_{l_{z}}$, from $k \leq k_{z},(3.2$ ) and (3.3), 
we have $2^{j_{z}}|\gamma(t)| \leq 2^{-k}$, which further implies (3.6). Noting $\frac{\gamma(t)}{t}$ is increasing on $\mathbb{R}^{+},(3.2)$ and (3.3), we then bound (3.5) by

$$
\begin{aligned}
& \sum_{n \in \mathbb{N}} \sum_{k \leq k_{z}} \int_{-\infty}^{\infty} \int_{|w| \leq 2^{-k+n}}\left|f\left(x_{1}-2^{i_{z}} t, x_{2}-w\right)\right| \cdot 2^{j_{z}}|\gamma(t)| 2^{2 k} 2^{-2 n} \mathrm{~d} w \phi_{l_{z}}(t) \frac{\mathrm{d} t}{|t|} \\
& \lesssim \sum_{n \in \mathbb{N}} 2^{-n} \sum_{k \leq k_{z}} 2^{k} 2^{j_{z}} \gamma\left(2^{l_{z}}\right) \frac{1}{2^{l_{z}}} \int_{|t| \lesssim 2^{l_{z}}} \frac{1}{2^{-k+n}} \int_{|w| \leq 2^{-k+n}}\left|f\left(x_{1}-2^{i_{z}} t, x_{2}-w\right)\right| \mathrm{d} w \mathrm{~d} t \\
& \lesssim M^{(1)} M^{(2)} f\left(x_{1}, x_{2}\right) \text {. }
\end{aligned}
$$

From the $L^{p}(\mathbb{R})$-boundedness of $M^{(1)}$ and $M^{(2)}$, which trivially leads to the estimate

$$
\left\|M_{I I_{a}, l_{z}}^{i_{z}, j_{z}} f-\mathbb{M}_{I I_{a}, l_{z}}^{i_{z}, j_{z}} f\right\|_{L^{p}\left(\mathbb{R}^{2}\right)} \lesssim\|f\|_{L^{p}\left(\mathbb{R}^{2}\right)}
$$

for all $p \in(1, \infty)$. This, combined with $(3.4)$, leads to

$$
\left\|M_{I I_{a}, l_{z}}^{i_{z}, j_{z}} f\right\|_{L^{p}\left(\mathbb{R}^{2}\right)} \lesssim\|f\|_{L^{p}\left(\mathbb{R}^{2}\right)}
$$

for all $p \in(1, \infty)$.

Consider $M_{I I_{b}, l_{z}}^{i_{z}, j_{z}} f$. We rewrite

$$
M_{I I_{b}, l_{z}}^{i_{z}, j_{z}} f=\sum_{k>0} M_{I I, l_{z}}^{i_{z}, j_{z}} P_{k+k_{z}}^{(2)} f=\sum_{m \in \mathbb{Z}} \sum_{k>0} M_{I I, l_{z}}^{i_{z}, j_{z}} P_{m}^{(1)} P_{k+k_{z}}^{(2)} f .
$$

For these $i_{z}, l_{z} \in \mathbb{Z}$, we let $m_{z}: \mathbb{R}^{2} \rightarrow \mathbb{R}$ be a measurable function satisfying

$$
2^{m_{z}} 2^{i_{z}} 2^{l_{z}}=1
$$

Then, we can split

$$
\begin{aligned}
M_{I I_{b}, l_{z}}^{i_{z}, j_{z}} f & =\sum_{m \leq m_{z}} \sum_{k>0} M_{I I, l_{z}}^{i_{z}, j_{z}} P_{m}^{(1)} P_{k+k_{z}}^{(2)} f+\sum_{m>m_{z}} \sum_{k>0} M_{I I, l_{z}}^{i_{z}, j_{z}} P_{m}^{(1)} P_{k+k_{z}}^{(2)} f \\
& =: M_{I I_{b}^{1}, l_{z}}^{i_{z}, j_{z}} f+M_{I I_{b}^{2}, l_{z}}^{i_{z}, j_{z}} f .
\end{aligned}
$$

Consider $M_{I I_{b}^{1}, l_{z}}^{i_{z}, j_{z}} f$. As in $M_{I I_{a}, l_{z}}^{i_{z}, j_{z}} f$, we will compare it with

$$
\mathbb{M}_{I I_{b}^{1}, l_{z}}^{i_{z}, j_{z}} f\left(x_{1}, x_{2}\right):=\sum_{m \leq m_{z}} \sum_{k>0} \int_{-\infty}^{\infty} P_{m}^{(1)} P_{k+k_{z}}^{(2)} f\left(x_{1}, x_{2}-2^{j_{z}} \gamma(t)\right) \phi_{l_{z}}(t) \frac{\mathrm{d} t}{|t|}
$$

After changing of variable $2^{j_{z}} \gamma(t)=: w$, we use (3.1) and the fact that $\left(\gamma^{-1}\right)^{\prime}(t) \gamma^{\prime}\left(\gamma^{-1}(t)\right)=$ 1 to establish

$$
\frac{1}{\gamma^{\prime}\left(\gamma^{-1}\left(w / 2^{j_{z}}\right)\right)}=\frac{\gamma\left(\gamma^{-1}\left(w / 2^{j_{z}}\right)\right)}{\gamma^{\prime}\left(\gamma^{-1}\left(w / 2^{j_{z}}\right)\right) \gamma^{-1}\left(w / 2^{j_{z}}\right)} \frac{\gamma^{-1}\left(w / 2^{j_{z}}\right)}{w / 2^{j_{z}}} \lesssim \frac{\gamma^{-1}\left(w / 2^{j_{z}}\right)}{w / 2^{j_{z}}}
$$


Then we have the following pointwise estimate

$$
\left|\mathbb{M}_{I I_{b}^{1}, l_{z}}^{i_{z}, j_{z}} f\left(x_{1}, x_{2}\right)\right| \lesssim M^{(2)}\left(\sum_{m \leq m_{z}} \sum_{k>0} P_{m}^{(1)} P_{k+k_{z}}^{(2)} f\right)\left(x_{1}, x_{2}\right),
$$

which trivially leads to

$$
\left\|\mathbb{M}_{I I_{b}^{1}, l_{z}}^{i_{z}, j_{z}} f\right\|_{L^{p}\left(\mathbb{R}^{2}\right)} \lesssim\|f\|_{L^{p}\left(\mathbb{R}^{2}\right)}
$$

for all $p \in(1, \infty)$. As the difference between $M_{I I_{a}, l_{z}}^{i_{z}, j_{z}} f(z)$ and $\mathbb{M}_{I I_{a}, l_{z}}^{i_{z}, j_{z}} f(z)$, we can bound $\left|M_{I I_{b}^{1}, l_{z}}^{i_{z}, j_{z}} f(z)-\mathbb{M}_{I I_{b}^{1}, l_{z}}^{i_{z}, j_{z}} f(z)\right|$ by $M^{(1)} M^{(2)}\left(\sum_{k>0} P_{k+k_{z}}^{(2)} f\right)(z)$, which further implies the $L^{p}(\mathbb{R})$-boundedness of the difference between $M_{I I_{b}^{1}, l_{z}}^{i_{z}, j_{z}} f(z)$ and $\mathbb{M}_{I I_{b}^{1}, l_{z}}^{i_{z}, j_{z}} f(z)$. Hence, from (3.7), we get

$$
\left\|M_{I I_{b}^{1}, l_{z}}^{i_{z}, j_{z}} f\right\|_{L^{p}\left(\mathbb{R}^{2}\right)} \lesssim\|f\|_{L^{p}\left(\mathbb{R}^{2}\right)}
$$

for all $p \in(1, \infty)$.

Consider $M_{I I_{b}^{2}, l_{z}}^{i_{z}, j_{z}} f$. We rewrite

$$
M_{I I_{b}^{2}, l_{z}}^{i_{z}, j_{z}} f=\sum_{m>0} \sum_{k>0} M_{I I, l_{z}}^{i_{z}, j_{z}} P_{m+m_{z}}^{(1)} P_{k+k_{z}}^{(2)} f .
$$

By interpolation, it suffices to prove that there exists a positive constant $\delta$ such that

$$
\left\|M_{I I, l_{z}}^{i_{z}, j_{z}} P_{m+m_{z}}^{(1)} P_{k+k_{z}}^{(2)} f\right\|_{L^{p}\left(\mathbb{R}^{2}\right)} \lesssim 2^{-\delta(m+k)}\|f\|_{L^{p}\left(\mathbb{R}^{2}\right)}
$$

for all $p \in[2, \infty)$, and

$$
\left\|M_{I I, l_{z}}^{i_{z}, j_{z}} P_{m+m_{z}}^{(1)} P_{k+k_{z}}^{(2)} f\right\|_{L^{p}\left(\mathbb{R}^{2}\right)} \lesssim\|f\|_{L^{p}\left(\mathbb{R}^{2}\right)}
$$

for all $p \in(1,2)$.

Proof of (3.8). The expression inside the $L^{p}\left(\mathbb{R}^{2}\right)$-norm on the left-hand side of (3.8) can be dominated by

$$
\left(\sum_{M, K \in \mathbb{Z}}\left|M_{I I, l_{z}}^{i_{z}, j_{z}} P_{m+M}^{(1)} P_{k+K}^{(2)} f\right|^{p}\right)^{1 / p},
$$

where $2^{M} 2^{i_{z}} 2^{l_{z}}=1$ and $2^{K} 2^{j_{z}} \gamma\left(2^{l_{z}}\right)=1$. Noting that

$$
\begin{aligned}
\left\|\left(\sum_{M, K \in \mathbb{Z}}\left|P_{m+M}^{(1)} P_{k+K}^{(2)} f\right|^{p}\right)^{1 / p}\right\|_{L^{p}\left(\mathbb{R}^{2}\right)} & \lesssim\left\|\left(\sum_{M, K \in \mathbb{Z}}\left|P_{m+M}^{(1)} P_{k+K}^{(2)} f\right|^{2}\right)^{1 / 2}\right\|_{L^{p}\left(\mathbb{R}^{2}\right)} \\
& \lesssim\|f\|_{L^{p}\left(\mathbb{R}^{2}\right)}
\end{aligned}
$$

for all $p \in[2, \infty)$, and the $l^{p}\left(\mathbb{Z}^{2}\right)$ and $L^{p}\left(\mathbb{R}^{2}\right)$ norms commute, we prove only

$$
\left\|M_{I I, l_{z}}^{i_{z}, j_{z}} P_{m+M}^{(1)} P_{k+K}^{(2)} f\right\|_{L^{p}\left(\mathbb{R}^{2}\right)} \lesssim 2^{-\delta(m+k)}\|f\|_{L^{p}\left(\mathbb{R}^{2}\right)}
$$


for all $p \in[2, \infty)$. Furthermore, if we bound the expression inside the $L^{p}\left(\mathbb{R}^{2}\right)$-norm on the left-hand side of 3.10 by

$$
\left(\sum_{l \in \mathbb{Z}}\left|M_{I I, l}^{i, j} P_{m+M}^{(1)} P_{k+K}^{(2)} f\right|^{p}\right)^{1 / p}
$$

where $2^{M} 2^{i} 2^{l}=1$ and $2^{K} 2^{j} \gamma\left(2^{l}\right)=1$, it is enough to show

$$
\left\|M_{I I, l}^{i, j} P_{m+M}^{(1)} P_{k+K}^{(2)} f\right\|_{L^{p}\left(\mathbb{R}^{2}\right)} \lesssim 2^{-\delta(m+k)}\|f\|_{L^{p}\left(\mathbb{R}^{2}\right)}
$$

for all $p \in[2, \infty)$. By Minkowski's inequality, then the following identity is valid,

$$
\left\|M_{I I, l}^{i, j} P_{m+M}^{(1)} P_{k+K}^{(2)} f\right\|_{L^{p}\left(\mathbb{R}^{2}\right)} \lesssim\|f\|_{L^{p}\left(\mathbb{R}^{2}\right)}
$$

for all $p \in(1, \infty)$. Therefore, by interpolating with $(3.12)$, to prove 3.11 we need to show the following estimate

$$
\left\|M_{I I, l}^{i, j} P_{m+M}^{(1)} P_{k+K}^{(2)} f\right\|_{L^{2}\left(\mathbb{R}^{2}\right)} \lesssim 2^{-\delta(m+k)}\|f\|_{L^{2}\left(\mathbb{R}^{2}\right)} .
$$

To get 3.13$)$, let us set $\Gamma_{l}(t):=\frac{\gamma\left(2^{l} t\right)}{\gamma\left(2^{l}\right)}$, and rewrite

$$
M_{I I, l}^{i, j} P_{m+M}^{(1)} P_{k+K}^{(2)} f\left(x_{1}, x_{2}\right)=\int_{-\infty}^{\infty} P_{m+M}^{(1)} P_{k+K}^{(2)} f\left(x_{1}-2^{i} 2^{l} t, x_{2}-2^{j} \gamma\left(2^{l}\right) \Gamma_{l}(t)\right) \phi(t) \frac{\mathrm{d} t}{|t|} .
$$

Furthermore, let

$$
T^{l} f\left(x_{1}, x_{2}\right):=\int_{-\infty}^{\infty} f\left(x_{1}-t, x_{2}-\Gamma_{l}(t)\right) \phi(t) \frac{\mathrm{d} t}{|t|},
$$

and for any given real numbers $a, b$, define

$$
\Delta_{a, b} f\left(x_{1}, x_{2}\right):=f\left(2^{a} x_{1}, 2^{b} x_{2}\right) .
$$

Therefore, it is easy to see that

$$
M_{I I, l}^{i, j} P_{m+M}^{(1)} P_{k+K}^{(2)} f=\Delta_{-i-l,-j-\log _{2} \gamma\left(2^{l}\right)} T^{l} \Delta_{i+l, j+\log _{2} \gamma\left(2^{l}\right)} P_{m+M}^{(1)} P_{k+K}^{(2)} f .
$$

Note that $2^{M} 2^{i} 2^{l}=1$ and $2^{K} 2^{j} \gamma\left(2^{l}\right)=1$, then

$$
\begin{aligned}
\Delta_{i+l, j+\log _{2} \gamma\left(2^{l}\right)} P_{m+M}^{(1)} P_{k+K}^{(2)} f & =P_{m+M+i+l}^{(1)} P_{k+K+j+\log _{2} \gamma\left(2^{l}\right)}^{(2)} \Delta_{i+l, j+\log _{2} \gamma\left(2^{l}\right)} f \\
& =P_{m}^{(1)} P_{k}^{(2)} \Delta_{i+l, j+\log _{2} \gamma\left(2^{l}\right)} f .
\end{aligned}
$$

So we can write

$$
M_{I I, l}^{i, j} P_{m+M}^{(1)} P_{k+K}^{(2)} f=\Delta_{-i-l,-j-\log _{2} \gamma\left(2^{l}\right)} T^{l} P_{m}^{(1)} P_{k}^{(2)} \Delta_{i+l, j+\log _{2} \gamma\left(2^{l}\right)} f .
$$


Since conjugation by $\Delta_{a, b}$ is an $L^{p}\left(\mathbb{R}^{2}\right)$ isometry, we then have

$$
\left\|M_{I I, l}^{i, j} P_{m+M}^{(1)} P_{k+K}^{(2)} f\right\|_{L^{2}\left(\mathbb{R}^{2}\right)}=\left\|T^{l} P_{m}^{(1)} P_{k}^{(2)} f\right\|_{L^{2}\left(\mathbb{R}^{2}\right)} .
$$

Let the multiplier of $T^{l} P_{m}^{(1)} P_{k}^{(2)} f$ be

$$
m\left(\xi_{1}, \xi_{2}\right):=\psi_{m}\left(\xi_{1}\right) \psi_{k}\left(\xi_{2}\right) \int_{-\infty}^{\infty} e^{-i \xi_{1} t-i \xi_{2} \Gamma_{l}(t)} \phi(t) \frac{\mathrm{d} t}{|t|}
$$

and the corresponding phase function be $\Phi(t):=-\xi_{1} t-\xi_{2} \Gamma_{l}(t)$, so

$$
\Phi^{\prime}(t)=-\xi_{1}-\xi_{2} \Gamma_{l}^{\prime}(t) \text { and } \Phi^{\prime \prime}(t)=-\xi_{2} \Gamma_{l}^{\prime \prime}(t) .
$$

We now consider the following two cases:

$\star$ If $\left|\xi_{1}\right| \geq 4 C_{1}^{\prime} e^{C_{1}^{\prime}}\left|\xi_{2}\right|$, from (3.1) and (3.2), this implies $\left|\Gamma_{l}^{\prime}(t)\right| \leq 2 C_{1}^{\prime} e^{C_{1}^{\prime}}$ with a bound independent of $l$, which further leads to $\left|\Phi^{\prime}(t)\right| \geq\left|\xi_{1}\right|-\left|\xi_{2} \Gamma_{l}^{\prime}(t)\right| \geq \frac{\left|\xi_{1}\right|}{2}$. Noting that $\xi_{1} \in \operatorname{supp} \psi_{m}$, we have $\left|\Phi^{\prime}(t)\right| \gtrsim 2^{m}$. This, by van der Corput's lemma and the fact that $\Phi^{\prime}(t)$ is monotonic, implies $\left|m\left(\xi_{1}, \xi_{2}\right)\right| \lesssim 2^{-m}$. Furthermore, by $\xi_{2} \in \operatorname{supp} \psi_{k}$ and $\left|\xi_{1}\right| \geq 4 C_{1}^{\prime} e^{C_{1}^{\prime}}\left|\xi_{2}\right|$, we have $\left|m\left(\xi_{1}, \xi_{2}\right)\right| \lesssim 2^{-\frac{1}{2}(m+k)}$. Applying Plancherel's theorem, we obtain (3.13) with $\delta=1 / 2$.

$\star$ If $\left|\xi_{1}\right| \leq 4 C_{1}^{\prime} e^{C_{1}^{\prime}}\left|\xi_{2}\right|$, from Theorem 1.3(ii), (3.1) and 3.2 , we have $\left|\Gamma_{l}^{\prime \prime}(t)\right| \geq$ $\frac{C_{2}\left(C_{2}+1\right)}{4 e^{C_{1}^{\prime}}}\left|\xi_{2}\right|$ with a bound independent of $l$. This, combined with the fact that $\xi_{2} \in \operatorname{supp} \psi_{k}$, leads to $\left|\Phi^{\prime \prime}(t)\right| \gtrsim 2^{k}$. We then apply van der Corput's lemma to obtain $\left|m\left(\xi_{1}, \xi_{2}\right)\right| \lesssim 2^{-k / 2}$. As in the first case, by Plancherel's theorem again, we obtain 3.13 with $\delta=1 / 4$.

Putting things together, we obtain 3.13 with $\delta=1 / 4$, this finishes the proof of (3.8).

Proof of (3.9). It suffices to show

$$
\left\|\left(\sum_{M, K \in \mathbb{Z}}\left|M_{I I, l_{z}}^{i_{z}, j_{z}} P_{m+M}^{(1)} P_{k+K}^{(2)} f\right|^{2}\right)^{1 / 2}\right\|_{L^{p}\left(\mathbb{R}^{2}\right)} \lesssim\left\|\left(\sum_{M, K \in \mathbb{Z}}\left|P_{m+M}^{(1)} P_{k+K}^{(2)} f\right|^{2}\right)^{1 / 2}\right\|_{L^{p}\left(\mathbb{R}^{2}\right)}
$$

for all $p \in(1,2)$, where $2^{M} 2^{i_{z}} 2^{l_{z}}=1$ and $2^{K} 2^{j_{z}} \gamma\left(2^{l_{z}}\right)=1$. We consider the more general estimate

$\left\|\left(\sum_{M, K \in \mathbb{Z}}\left|M_{I I, l_{z}}^{i_{z}, j_{z}} P_{m+M}^{(1)} P_{k+K}^{(2)} f\right|^{p_{1}}\right)^{1 / p_{1}}\right\|_{L^{p_{2}\left(\mathbb{R}^{2}\right)}} \lesssim\left\|\left(\sum_{M, K \in \mathbb{Z}}\left|P_{m+M}^{(1)} P_{k+K}^{(2)} f\right|^{p_{1}}\right)^{1 / p_{1}}\right\|_{L^{p_{2}\left(\mathbb{R}^{2}\right)}}$

for certain $p_{1} \in(1, \infty]$ and $p_{2} \in(1, \infty)$. 
For the case of $p_{1}=\infty$ and $p_{2}=2$, by linearization, we have that the expression inside the $L^{p_{2}}\left(\mathbb{R}^{2}\right)$-norm on the left-hand side of 3.15 can be bounded by $M_{I I, \gamma}\left(\sup _{M, K \in \mathbb{Z}}\right.$ $\left.\left|P_{m+M}^{(1)} P_{k+K}^{(2)} f\right|\right)$. It is clear that $(3.8)$ implies the $L^{2}\left(\mathbb{R}^{2}\right)$-boundedness of $M_{I I, \gamma}$. Therefore, (3.15) holds for $p_{1}=\infty$ and $p_{2}=2$.

For the case of $p_{1}=p_{2}$ and $p_{1}, p_{2} \in(1, \infty)$, by replacing $f$ by $P_{m+M}^{(1)} P_{k+K}^{(2)} f$, we have that 3.15 is equivalent to

$$
\left\|M_{I I, l_{z}}^{i_{z}, j_{z}} P_{m+M}^{(1)} P_{k+K}^{(2)} f\right\|_{L^{p}\left(\mathbb{R}^{2}\right)} \lesssim\|f\|_{L^{p}\left(\mathbb{R}^{2}\right)}
$$

for all $p \in(1, \infty)$. We admit it first, which proof will be submit later. Then the interpolation argument implies that (3.14) holds for all $p \in(4 / 3,2)$. Repeating the interpolation argument and using $p_{1}=\infty$ and $p_{2} \in(4 / 3,2)$, we can prove that 3.14 holds for all $p \in(8 / 7,2)$. Reiterating this process sufficiently many times we can show that 3.14 holds for all $p \in(1,2)$.

Therefore, it suffices to show 3.16 . Furthermore, note that $M_{I I, l_{z}}^{i_{z}, j_{z}} P_{m+M}^{(1)} P_{k+K}^{(2)} f$ can be bounded by $M_{I I, \gamma} M^{(1)} M^{(2)} f$, and the $L^{p}\left(\mathbb{R}^{2}\right)$-boundedness of $M_{I I, \gamma}, M^{(1)}$ and $M^{(2)}$ for all $p \in[2, \infty)$, thus we may restrict $p \in(1,2)$ in the proof of $(3.16)$. This further reduces to showing

$$
\left\|\left(\sum_{l \in \mathbb{Z}}\left|M_{I I, l}^{i, j} P_{m+M}^{(1)} P_{k+K}^{(2)} f\right|^{2}\right)^{1 / 2}\right\|_{L^{p}\left(\mathbb{R}^{2}\right)} \lesssim\left\|\left(\sum_{l \in \mathbb{Z}}\left|P_{m+M}^{(1)} f\right|^{2}\right)^{1 / 2}\right\|_{L^{p}\left(\mathbb{R}^{2}\right)}
$$

for all $p \in(1,2)$, where $2^{M} 2^{i} 2^{l}=1$ and $2^{K} 2^{j} \gamma\left(2^{l}\right)=1$. Repeating the method of bootstrapping an iterated interpolation argument above, it suffices to prove

$$
\left\|M_{I I, l}^{i, j} P_{m+M}^{(1)} P_{k+K}^{(2)} f\right\|_{L^{p}\left(\mathbb{R}^{2}\right)} \lesssim\|f\|_{L^{p}\left(\mathbb{R}^{2}\right)}
$$

for all $p \in(1, \infty)$, which can be followed from Minkowski's inequality. We then obtain (3.9) and complete the proof of Theorem 1.3 .

\section{Acknowledgments}

The author would like to thank the referee for carefully reading the manuscript and for offering valuable comments, and also Lixin Yan for the many helpful suggestions.

\section{References}

[1] N. Bez, $L^{p}$-boundedness for the Hilbert transform and maximal operator along a class of nonconvex curves, Proc. Amer. Math. Soc. 135 (2007), no. 1, 151-161.

[2] J. Bourgain, Averages in the plane over convex curves and maximal operators, J. Analyse Math. 47 (1986), 69-85. 
[3] A. Carbery, Differentiation in lacunary directions and an extension of the Marcinkiewicz multiplier theorem, Ann. Inst. Fourier (Grenoble) 38 (1988), no. 1, $157-168$.

[4] A. Carbery, M. Christ, J. Vance, S. Wainger and D. K. Watson, Operators associated to flat plane curves: $L^{p}$ estimates via dilation methods, Duke Math. J. 59 (1989), no. $3,675-700$.

[5] A. Carbery, J. Vance, S. Wainger and D. Watson, The Hilbert transform and maximal function along flat curves, dilations, and differential equations, Amer. J. Math. 116 (1994), no. 5, 1203-1239.

[6] A. Carbery and S. Ziesler, Hilbert transforms and maximal functions along rough flat curves, Rev. Mat. Iberoamericana 10 (1994), no. 2, 379-393.

[7] H. Carlsson, M. Christ, A. Córdoba, J. Duoandikoetxea, J. L. Rubio de Francia, J. Vance, S. Wainger and D. Weinberg, $L^{p}$ estimates for maximal functions and Hilbert transforms along flat convex curves in $\mathbb{R}^{2}$, Bull. Amer. Math. Soc. (N.S.) 14 (1986), no. 2, 263-267.

[8] H. Carlsson and S. Wainger, Maximal functions related to convex polygonal lines, Indiana Univ. Math. J. 34 (1985), no. 4, 815-823.

[9] A. Córdoba and R. Fefferman, On differentiation of integrals, Proc. Nat. Acad. Sci. U.S.A. 74 (1977), no. 6, 2211-2213.

[10] A. Córdoba and J. L. Rubio de Francia, Estimates for Wainger's singular integrals along curves, Rev. Mat. Iberoamericana 2 (1986), no. 1-2, 105-117.

[11] C. Demeter and F. Di Plinio, Logarithmic $L^{p}$ bounds for maximal directional singular integrals in the plane, J. Geom. Anal. 24 (2014), no. 1, 375-416.

[12] S. Guo, J. Hickman, V. Lie and J. Roos, Maximal operators and Hilbert transforms along variable non-flat homogeneous curves, Proc. Lond. Math. Soc. (3) 115 (2017), no. $1,177-219$.

[13] S. Guo, J. Roos, A. Seeger and P.-L. Yung, A maximal function for families of Hilbert transforms along homogeneous curves, Math. Ann. 377 (2020), no. 1-2, 69-114.

[14] K. Hare and F. Ricci, Maximal functions with polynomial densities in lacunary directions, Trans. Amer. Math. Soc. 355 (2003), no. 3, 1135-1144.

[15] S. Hong, J. Kim and C. W. Yang, Maximal operators associated with vector polynomials of lacunary coefficients, Canad. J. Math. 61 (2009), no. 4, 807-827. 
[16] A. Iosevich, Maximal operators associated to families of flat curves in the plane, Duke Math. J. 76 (1994), no. 2, 633-644.

[17] G. A. Karagulyan, On unboundedness of maximal operators for directional Hilbert transforms, Proc. Amer. Math. Soc. 135 (2007), no. 10, 3133-3141.

[18] I. Łaba, A. Marinelli and M. Pramanik, On the maximal directional Hilbert transform, Anal. Math. 45 (2019), no. 3, 535-568.

[19] W. Li, Maximal functions associated with nonisotropic dilations of hypersurfaces in $\mathbb{R}^{3}$, J. Math. Pures Appl. (9) 113 (2018), 70-140.

[20] G. Marletta, Maximal functions with mitigating factors in the plane, J. London Math. Soc. (2) 59 (1999), no. 2, 647-656.

[21] G. Marletta and F. Ricci, Two-parameter maximal functions associated with homogeneous surfaces in $\mathbb{R}^{n}$, Studia Math. 130 (1998), no. 1, 53-65.

[22] A. Nagel, N. Riviere and S. Wainger, A maximal function associated to the curve $\left(t, t^{2}\right)$, Proc. Nat. Acad. Sci. U.S.A. 73 (1976), no. 5, 1416-1417.

[23] A. Nagel, E. M. Stein and S. Wainger, Differentiation in lacunary directions, Proc. Nat. Acad. Sci. U.S.A. 75 (1978), no. 3, 1060-1062.

[24] A. Nagel, J. Vance, S. Wainger and D. Weinberg, Hilbert transforms for convex curves, Duke Math. J. 50 (1983), no. 3, 735-744.

[25] _ Maximal functions for convex curves, Duke Math. J. 52 (1985), no. 3, 715722.

[26] W. C. Nestlerode, Singular integrals and maximal functions associated with highly monotone curves, Trans. Amer. Math. Soc. 267 (1981), no. 2, 435-444.

[27] E. M. Stein, Maximal functions II: Homogeneous curves, Proc. Nat. Acad. Sci. U.S.A. 73 (1976), no. 7, 2176-2177.

[28] E. M. Stein and S. Wainger, Maximal functions associated to smooth curves, Proc. Nat. Acad. Sci. U.S.A. 73 (1976), no. 12, 4295-4296.

[29] - Problems in harmonic analysis related to curvature, Bull. Amer. Math. Soc. 84 (1978), no. 6, 1239-1295.

[30] J.-O. Strömberg, Weak estimates on maximal functions with rectangles in certain directions, Ark. Mat. 15 (1977), no. 2, 229-240. 
[31] J. Vance, S. Wainger and J. Wright, The Hilbert transform and maximal function along nonconvex curves in the plane, Rev. Mat. Iberoamericana 10 (1994), no. 1, 93-121.

[32] J. Wright, $L^{p}$ estimates for operators associated to oscillating plane curves, Duke Math. J. 67 (1992), no. 1, 101-157.

[33] S. Ziesler, $L^{p}$-boundedness of the Hilbert transform and maximal function associated to flat plane curves, Proc. Amer. Math. Soc. 122 (1994), no. 4, 1035-1043.

Haixia $\mathrm{Yu}$

Department of Mathematics, Shantou University, Shantou, 515063, China

E-mail address: yuhx26@mail.sysu.edu.cn 